\title{
3-D B-spline Wavelet-Based Local Standard Deviation (BWLSD): Its Application to Edge Detection and Vascular Segmentation in Magnetic Resonance Angiography
}

\author{
Zhenyu He • Albert C.S. Chung
}

Received: 11 September 2008 / Accepted: 26 May 2009 / Published online: 22 July 2009

(C) Springer Science+Business Media, LLC 2009

\begin{abstract}
Extracting reliable image edge information is crucial for active contour models as well as vascular segmentation in magnetic resonance angiography (MRA). However, conventional edge detection techniques, such as gradientbased methods and wavelet-based methods, are incapable of returning reliable detection responses from low contrast edges in the images. In this paper, we propose a novel edge detection method by combining B-spline wavelet magnitude with standard deviation inside local region. It is proved theoretically and demonstrated experimentally in this paper that the new edge detection method, namely BWLSD, is able to give consistent and reliable strengths for edges with different image contrasts. Moreover, the relationship between the size of local region with non-zero wavelet magnitudes and the scale of wavelet function is established. This relationship indicates that if the scale of the adopted wavelet function is $s$, then the size of a local region, from which the standard deviation is estimated, should be $2 s-1$. The proposed edge detection technique is embedded in FLUX, namely, BWLSD-FLUX, for vascular segmentation in MRA image volumes. Experimental results on clinical images show that, as compared with the conventional FLUX, BWLSD-FLUX can achieve better segmentations of vasculatures in MRA images under same initial conditions.
\end{abstract}

\section{Z. He (凶) · A.C.S. Chung}

Lo Kwee-Seong Medical Image Analysis Laboratory, Department of Computer Science and Engineering, The Hong Kong University of Science and Technology, Hong Kong, Hong Kong e-mail: csezyhe@cse.ust.hk

A.C.S. Chung

e-mail: achung@cse.ust.hk
Keywords Magnetic resonance angiography (MRA) . Vascular segmentation · B-spline wavelet · Local standard deviation · FLUX

\section{Introduction}

Vascular diseases, covering diseases from arteries, veins, capillaries to lymph vessels, are the leading causes of death in China and Australia (He et al. 2005; AIHW 2004) and have also become one of the leading killers in the USA and most European countries (Rinkel et al. 1998; CDC 2008). Along with Digital Subtraction Angiography (DSA) and Computed Tomography Angiography (CTA), Magnetic Resonance Angiography (MRA) is one of the most widely used medical imaging modalities for visualizing three-dimensional (3-D) brain vascular structures in patients.

Image segmentation of blood vessels in 3-D angiographic images is capable of providing valuable, patient-specific information of blood vessels, such as shape, size, geometric relations between vessels, and thus plays a vital role in diagnosis and prognosis of vascular diseases. For example, using the degree of a stenosis or the size of an aneurysm, clinicians can judge the risk of stroke or aneurysm rupture and then design an appropriate treatment planning for the patients. Kirbas and Quek (2004) systematically reviewed vascular segmentation techniques proposed in the past decades and classified these techniques into the following categories: multi-scale approaches (Huang and Stockman 1993; Chwialkowski et al. 1996; Armande et al. 1999), skeleton-based approaches (Polli and Valli 1997; Yim et al. 2000; Sorantin et al. 2002), ridge-based approaches (Guo and Richardson 1998; Bullitt et al. 2001; Staal et al. 2004), region growing approaches (O'Brien and 
Ezquerra 1994; Higgins et al. 1996; Schmitt et al. 2002), differential geometry-based approaches (Prinet et al. 1995; Krissian et al. 1996), matching filters approaches (Chen et al. 1998; Sato et al. 1998; Hoover et al. 2000), mathematical morphology schemes (Figueiredo and Leitao 1995; Eiho and Qian 1997; Mendonca and Campilho 2006), active contour models (Kass et al. 1988; Malladi et al. 1995; Sethian 1996; Caselles et al. 1997; Xu and Prince 1998; Lorigo et al. 2001; Vasilevskiy and Siddiqi 2002), trackingbased approaches (Quek et al. 1999; Aylward and Bullitt 2002; Wong and Chung 2007), artificial intelligencebased approaches (Smets et al. 1988; Bombardier et al. 1997), neural network-based approaches (Rekovei and Sun 1995; Hunter et al. 1995) and tube-like object detection approaches (Parvin et al. 1994).

Active contour models are most popular among the above vascular segmentation techniques. Snakes were first introduced by Kass et al. (1988) for segmenting objects in images and have been widely used in medical segmentation tasks (Duncan and Ayache 2000). In Snakes, the initial boundary is deformed iteratively by minimizing an energy functional, which is determined by the image forces (external forces) as well as the smoothness of the curves (internal forces). It is well known that its performance depends on the boundary initialization and input image edge map and Snakes have difficulties in progressing into boundary concavities. Moreover, the convergence and stability of its deformation process may be unpredictable. To overcome these limitations, extensions of Snakes have been developed. Several methods have been developed to address the dependence of Snakes on initial boundary by increasing the capture range of the external force fields (Cohen and Cohen 1993; Leroy et al. 1996). Xu and Prince (1998) proposed a new external force, namely, gradient vector flow (GVF), to move Snakes into boundary concavities. Topologically adaptable surface (T-surface) is a variant of the classical Snakes (McInerney and Terzopoulos 2000). It has a better topologically adaptable property and is independent of deformable contour model parameterization. Curve evolution scheme, implemented by the level set method, is another kind of variants of classical Snakes, which uses partial differential equations (PDE) to govern boundary evolution (Caselles et al. 1997). This scheme is called geodesic active contour. Further, motivated by the discovery of more general level set equations for curvature-based evolution (Ambrosio and Soner 1996), CURVES algorithm, an extension of geodesic active contour, was proposed for vascular segmentation in MRA images (Lorigo et al. 2001). To segment narrow elongated vessels, FLUX was developed by Vasilevskiy and Siddiqi (2002). The key idea is to exploit vector direction along with its magnitude. To detect the boundary of thin blood vessels, geodesic active contour was mended by incorporating capillary effects (Yan and Kassim 2006) or combining a new edge alignment term (Holtzman-Gazit et al.
2006). A level-set-based geometric regularization method, which can minimize the leakage, was proposed by estimating the local orientation of the evolving front and then using it as shape induced information for anisotropic propagation (Gooya et al. 2008).

In active contour models, image edge information is important for precisely detecting blood vessel boundaries and extracting blood vessels. For example, contour evolution stops at positions where the values of gradient magnitude are large (Malladi et al. 1995). Geodesic active contour and T-snake also utilize image gradient magnitude to define the stopping criteria of contour evolution (Caselles et al. 1997; McInerney and Terzopoulos 2000). Due to bias fields, motion artifacts during image acquisition and complex blood flows inside vessels, intensity within the same tissue type can vary in MRA images. This causes inhomogeneous intensity pattern within the same tissue type and makes the intensity difference between vessels and background become small and varying. As such, the conventional methods for edge detection (e.g., gradient magnitude, Hessian matrixFrangi et al. 1998; Sato et al. 1998; wavelet-Mallat 1998) are difficult to provide a reliable edge map for MRA images because they are not specifically designed for dealing with the problem of intensity inhomogeneity. For instance, the gradient magnitude $|\nabla I|$ or $|\nabla G * I|$, where $G$ is a Gaussian filter, $I$ is the original image, $\nabla$ is a gradient operator and $*$ stands for convolution, cannot obtain a strong edge strength at the vessel boundary in a low intensity contrast image region. In deformable model, small edge strength of the real vessel boundary in a low intensity contrast region hardly generates adequate force to compete with other boundaries with a large edge strength. As a result, the propagating front/surface would not halt at the vessel boundary with small edge strength but would be attracted by edges of other unrelated tissues or even background noise. Consequently, a contour leakage or discontinuity is occurred. Dealing with intensity inhomogeneity of blood vessels in MRA images is not only a technological challenge but also a basic requirement for a robust algorithm on vascular segmentation.

In this paper, we propose a novel method against intensity inhomogeneity and noise for edge detection in MRA images. The basic idea is to combine B-spline wavelet magnitudes and their local standard deviations to remove the effects of intensity inhomogeneity and noise. Thus, this method is called B-spline wavelet-based local standard deviation (BWLSD). We prove that, via BWLSD, detected edges in regions with different intensity contrasts can give the same edge strength. BWLSD is also robust to noise due to the inherent advantage of wavelet transform on denoising. Though BWLSD is built on the framework of wavelet-based edge detection (Mallat and Zhong 1992; Mallat and Hwang 1992), it overcomes the limitation of 
the conventional wavelet-based edge detection techniques on smoothing out the intensity inhomogeneity. Certainly, the advantages of wavelet-based edge detection techniques are maintained in BWLSD. Although BWLSD is also motivated by the work of Tang et al. (2000), we do not characterize Dirac structure edges with wavelet transform. Instead, we present two lemmas in this paper on the setting of the size of a local region, from which the standard deviation is estimated, according to the scale of wavelet transform. In addition, Tang et al. handled 2-D images with slight noise and without intensity inhomogeneity, while we develop BLWSD for 3-D images, in which there are 3-D symmetric or asymmetric tube-like objects with noise and intensity inhomogeneity. It is noted that we propose criteria to determine the local region size for estimating the standard deviation of Bspline wavelet magnitudes. Therefore, in our work, the local region size is chosen according to our criteria but not experiments or experience. As compared with the gradient-based method, the most popular edge detector, BWLSD offers a better performance on edge detection because BWLSD can smooth out the intensity inhomogeneity while the gradientbased method cannot. In contrast to Hessian matrix-based technique, another commonly used approach for edge or centerline detection, BWLSD is independent of the shape and structures of edges and thus prior information about shape and structure is not needed for BWLSD.

The rest of this paper is organized as follows. In Sect. 2.1, we introduce the wavelet-based edge detection technique and discuss its limitations on dealing with the problem of intensity inhomogeneity. BWLSD is then presented in Sect. 2.2, where we propose and prove a lemma that the BWLSD values of detected edges in regions with different contrast levels remain constant. In Sect. 2.3, we theoretically establish the relationship between the scale of wavelet transform and the size of local region, from which the standard deviation of B-spline wavelet magnitudes is estimated. In Sect. 2.4, concepts and details about the FLUX model is given. Implementation issues are discussed in Sect. 3. Experimental results on synthetic and clinical data sets are presented in Sect. 4. The conclusion of this paper is made in Sect. 5. Finally, the proofs of lemmas proposed in this paper are given in the appendices.

\section{Methodology}

\subsection{Introduction to Edge Detection Using 3-D B-spline Wavelet Transform}

Let $f(\mathbf{x})$ represent the input image and $\theta(\mathbf{x})$ a smooth function satisfying three requirements: (1) $\hat{\theta}(\mathbf{0})=1$, (2) $\theta(\mathbf{x})=$ $\theta(-\mathbf{x})$, and (3) $\theta(\infty)=0$. Here, $\hat{\theta}$ is the Fourier transform of $\theta$ and this smooth function at scale $s$ is $\theta_{s}(\mathbf{x})$. Then the absolute local maxima of the first derivative of $f * \theta_{s}(\mathbf{x})$ are the points with sharp intensity transition within a local region (Mallat and Zhong 1992; Mallat and Hwang 1992). These points reflect the edges in one image. Quadratic B-spline function $\theta(x)$ (Wang and Lee 1998) is used as the smooth function, and is given as,

$\theta(x)= \begin{cases}8|x|^{3}-8|x|^{2}+\frac{4}{3}, & |x| \leq 0.5, \\ -\frac{8}{3}|x|^{3}+8|x|^{2}-8|x|+\frac{8}{3}, & 0.5 \leq|x| \leq 1, \\ 0, & 1 \leq|x| .\end{cases}$

Apparently, $\theta(x)$ is a function compactly supported within $[-1,1]$ and satisfies the three requirements mentioned above. $\psi(x)$, the first derivative of function $\theta(x)$, is given as,

$\psi(x)= \begin{cases}24 \frac{|x|^{3}}{x}-16 \frac{|x|^{2}}{x}, & |x| \leq 0.5, \\ -8 \frac{|x|^{3}}{x}+16 \frac{|x|^{2}}{x}-8 \frac{|x|}{x}, & 0.5 \leq|x| \leq 1, \\ 0, & 1 \leq|x|,\end{cases}$

and $\psi(x)$ satisfies

$\int_{R} \psi(x) d x=0$.

According to the definitions of wavelet function (Mallat $1998)$ and (3), $\psi(x)$ is a wavelet function. Therefore, $\psi(x)$ is called the corresponding wavelet function of the smooth function $\theta(x)$. The reason for choosing quadratic B-spline function instead of Gaussian function as the smooth function is that the quadratic B-spline function is both smooth and compactly supported. As such, the truncation error in numerical implementation can be minimized. Considering the 3 -D case, namely $\mathbf{x}=\left\{x_{1}, x_{2}, x_{3}\right\}$, the following wavelet functions are obtained

$\psi^{i}(\mathbf{x})=\frac{\partial}{\partial x_{i}} \theta(\mathbf{x}), \quad i=1,2,3$.

By introducing a scale parameter $s$, the smooth function $\theta(\mathbf{x})$ and the wavelet function $\psi(\mathbf{x})$ at scale $s$ are denoted as

$\theta_{s}(\mathbf{x})=\frac{1}{s^{3}} \theta\left(\frac{\mathbf{x}}{s}\right), \quad \psi_{s}(\mathbf{x})=\frac{1}{s^{3}} \psi\left(\frac{\mathbf{x}}{s}\right)$.

Consequently, the corresponding wavelet transforms at scale $s$ are given as

$W_{s}^{i} f(\mathbf{x})=\left(f * \psi_{s}^{i}\right)(\mathbf{x})=s \frac{\partial}{\partial x_{i}}\left(f * \theta_{s}\right)(\mathbf{x}), \quad i=1,2,3$.

It is easy to see that the gradient is

$\nabla W_{s} f(\mathbf{x})=\left(\begin{array}{l}W_{s}^{1} f(\mathbf{x}) \\ W_{s}^{2} f(\mathbf{x}) \\ W_{s}^{3} f(\mathbf{x})\end{array}\right)=s \nabla\left(f * \theta_{s}\right)(\mathbf{x})$. 
The magnitude of gradient $\nabla W_{s} f(\mathbf{x})$ is

$\left|\nabla W_{s} f(\mathbf{x})\right|=\sqrt{\sum_{i=1}^{3}\left(\left|W_{s}^{i} f(\mathbf{x})\right|^{2}\right)}$.

The local maxima of $\left|\nabla W_{s} f(\mathbf{x})\right|$ are the locations of the points with sharp intensity transitions. The direction of gradient $\nabla W_{s} f(\mathbf{x})$ is

$$
E=\left(\begin{array}{c}
\alpha \\
\beta \\
\gamma
\end{array}\right)=\left(\begin{array}{c}
\arccos \left(\frac{W_{s}^{1} f(\mathbf{x})}{\left|\nabla W_{s} f(\mathbf{x})\right|}\right) \\
\arccos \left(\frac{W_{s}^{2} f(\mathbf{x})}{\left|\nabla W_{s} f(\mathbf{x})\right|}\right) \\
\arccos \left(\frac{W_{s}^{3} f(\mathbf{x})}{\left|\nabla W_{s} f(\mathbf{x})\right|}\right)
\end{array}\right) .
$$

This wavelet-based technique can reliably detect the edges in an image without intensity inhomogeneity and noise, and does not require any prior edge shape information. For ex- ample, Fig. 1(b) gives the detected edges of an image, as shown in Fig. 1(a), in which there is a white rectangle lying inside a black background. From Fig. 1(b), all edges are satisfactorily detected. However, the performance of the wavelet-based technique can be adversely affected when intensity inhomogeneity exists in the image. For illustration, Fig. 1(c) is the other image with intensity inhomogeneity, in which the left region has low contrast while the right region has high contrast. The edges detected from Fig. 1(c) are shown in Fig. 1(d), where the edges in the left region have weaker strength than the edges in the right region. Further, if one image is contaminated by both intensity inhomogeneity and noise, the performance of edge detection by using the wavelet-based technique can become worse and even unacceptable. An image, as shown in Fig. 1(f), is generated by adding a Gaussian white noise into the image as shown in Fig. 1(c). The edges detected from Fig. 1(f) by using the
Fig. 1 Comparisons between the wavelet-based edge detection technique and BWLSD. (a) A 2-D binary image without intensity inhomogeneity and noise.

(b) Edges detected by using the wavelet-based technique.

(c) A 2-D image with intensity inhomogeneity but without noise. (d) Edges detected by using the wavelet-based technique. (e) Edges detected by using BWLSD. (f) A 2-D image with both intensity inhomogeneity and noise.

(g) Edges detected by using the wavelet-based technique.

(h) Edges detected by using BWLSD

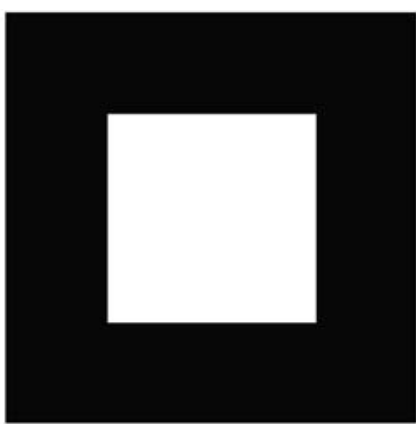

(a)

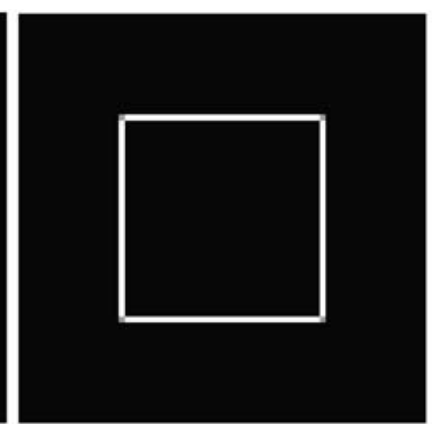

(b)

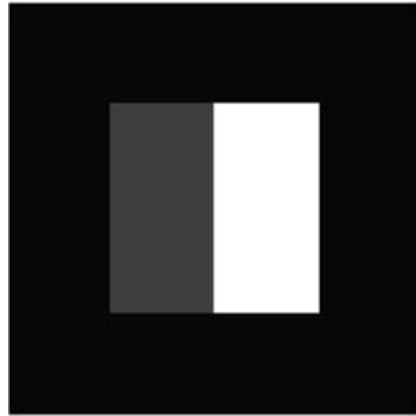

(c)

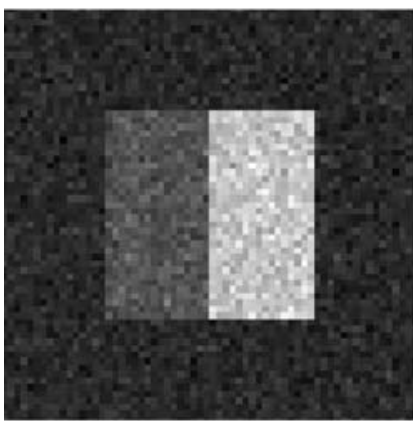

(f)

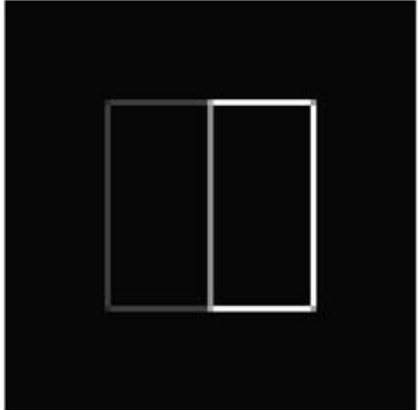

(d)

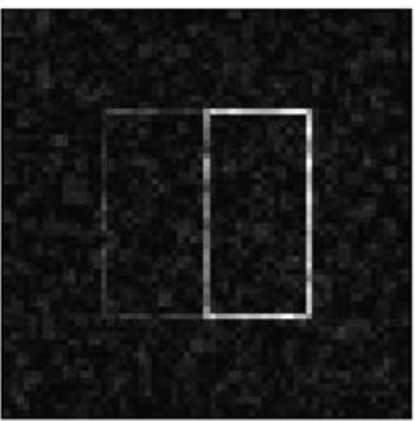

(g)

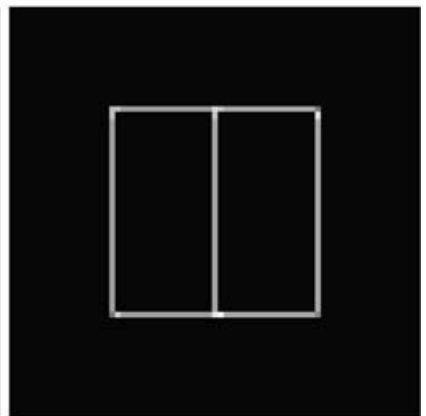

(e)

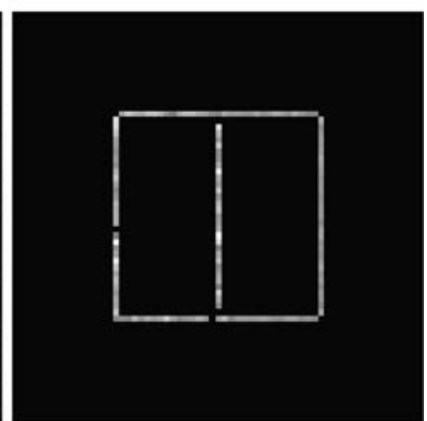

(h) 
wavelet-based technique are shown in Fig. $1(\mathrm{~g})$. From the figure, it is obvious that the edges in the left region are badly confused with the background and noise.

In this paper, we propose a new method based on a new edge measurement function, called B-spline wavelet-based local standard deviation (BWLSD). BWLSD is derived from the wavelet-based edge detection technique. This method is effective to cope with not only images with intensity inhomogeneity, as illustrated in Fig. 1(e), but also images with both intensity inhomogeneity and noise, as shown in Fig. 1(h).

\subsection{New Function to Measure Edge Strength}

For a 3-D image, based on local homogeneity of voxel intensity, one local image edge region $A$ can be statistically modeled as,

$f(A(\cdot)) \sim N\left(\mu_{A}, \sigma_{A}\right)$,

where mean value $\mu_{A}$ refers to the ideal luminosity value, and standard deviation $\sigma_{A}$ represents the inevitable luminosity variation introduced by the image acquisition devices. This is a reasonable assumption for mimicking the luminosity and contrast bias introduced by the image acquisition process. Another local image edge region $B$ with different contrast and luminosity in the same image can then be represented as

$f(B(\cdot))=K f(A(\cdot))+C, \quad K>0$.

Here, the terms $K$ and $C$ are variables reflecting the difference between the image edge regions $A$ and $B$ on intensity contrast and luminosity. Both $K$ and $C$ are space-dependent scale functions. In practice, they can be considered as constants when the size of one local image region $d$ is small enough. The size parameter $d$ can be treated as a bridge linking the scale of wavelet transform mentioned above and a local region of the 3-D image. It is important to select a proper value for $d$. If the value of $d$ is too large, several image regions with different contrast and luminosity will be falsely partitioned into one region and the above-mentioned statistical model is no longer precise. On the contrary, if the value of $d$ is too small, the number of voxels inside one local image edge region is not sufficient to provide stable statistical estimations. We will discuss this problem and present two related lemmas in the next subsections.

Without loss of generality, this statistical model $N\left(\mu_{A}, \sigma_{A}\right)$ can be further simplified into $N(0,1)$ by imposing $\mu_{A}=0$ and $\sigma_{A}=1$. This simplified assumption is acceptable to characterize any bias or amplification of luminosity and contrast drifts brought by the acquisition function (Foracchia et al. 2003). Consequently, the statistical description of the local image edge region $B$ is $f(B(\cdot)) \sim N(C, K)$.
By combining this statistical model and the waveletbased technique mentioned above, we develop a novel function $G$ to measure edge strengths in image edge regions with different intensity contrasts. This new measurement function is named as B-spline wavelet-based local standard deviation (BWLSD) function. For an edge point $\mathbf{x}$ belonging to a local region $R$, the BWLSD function is defined as

$G(\mathbf{x})=\frac{\left|\nabla W_{s} f(\mathbf{x})\right|}{\sigma\left(\left|\nabla W_{s} f(R)\right|\right)}$,

where $\left|\nabla W_{s} f(R)\right|$ is the collection of wavelet magnitudes of all points within $R$, namely, $\left|\nabla W_{s} f(R)\right|=\left\{\left|\nabla W_{s} f(\mathbf{x})\right| \mid\right.$ $\mathbf{x} \in R\}$, and $\sigma()$ represents the standard deviation of the B-spline wavelet magnitudes.

Consequently, for arbitrary points, $A$ and $B$, their measurements are given as

$G(A(\cdot))=\frac{\left|\nabla W_{s} f(A(\cdot))\right|}{\sigma\left(\left|\nabla W_{s} f(A)\right|\right)}$,

$G(B(\cdot))=\frac{\left|\nabla W_{s} f(B(\cdot))\right|}{\sigma\left(\left|\nabla W_{s} f(B)\right|\right)}$.

We mathematically prove that the BWLSD values of two image edges with different contrast and luminosity are the same.

Lemma 1 The BWLSD values of two image edges with different contrasts and luminosities are equal.

The proof of Lemma 1 is given in Appendix A. Lemma 1 reveals that the BWLSD value of one edge keeps constant regardless of its contrast and luminosity. That is, BWLSD can return a strong strength from the edge with low contrast, as will be demonstrated in the experimental results section. This property is also very useful for dealing with the problem of contour leakage or discontinuity during active contour evolution.

2.3 Relationship Between the Scale of Wavelet Transform and the Size of Local Region

In the previous subsection, to tackle the problem of image intensity inhomogeneity, we have proposed a novel measurement function BWLSD based on B-spline wavelet function and local standard deviation of wavelet magnitudes. We have also pointed out that the choice of local size $d$ is a crucial issue, which inevitably influences the final measurement result. As far as we know, though a large number of techniques making use of the local image properties have been proposed for image segmentation and contrast enhancement, most of them set the value for local region size empirically. In this subsection, we mathematically discuss the problem of choosing a proper value for local region size and illustrate the relationship between the scale of B-spline wavelet 
transform and the size of local region. Two lemmas are further proposed and proved in this discussion. It is assumed that a vessel is approximately regarded as a 3-D symmetric tube. Hence, we first discuss the case of 3-D symmetric tube.

Lemma 2 For a symmetric 3-D tube with radius $r$, the size of local region with non-zero wavelet magnitude is $2 s-1$ on each side of the tube regardless of the direction and shape of the tube. $s$ is the scale of B-spline wavelet transform.

The proof of Lemma 2 is given in Appendix B. On the basis of Lemma 2, the width of local region with non-zero wavelet magnitudes only depends on the scale of wavelet function while the width is independent of the radius of the 3 -D tube. In a discrete image, the true edge is described by the points as close as possible but not exactly located at the center of the true edge in theory. As such, the wavelet transform not only detects the edge but also shifts the edge into an edge region around the center of the true edge, which is determined by the wavelet scale. The region with non-zero wavelet magnitudes can be viewed as the edge region and the region with zero wavelet magnitudes can be viewed as the non-edge region. Since the points within an edge/nonedge region are of the same local statistical property and in general the size of an edge region is smaller than that of a non-edge region, it is therefore reasonable to choose the size of edge region as the size of local region for estimating the local deviation of B-spline wavelet magnitudes.

Of course, not all vessels can be considered as 3-D symmetric tube. For example, the vessel junction is not symmetric. Therefore, Lemma 2 is extended to the situation of 3-D asymmetric tube to deal with this case.

Lemma 3 For an asymmetric 3-D tube with radii $r$, the size of region with non-zero wavelet magnitude is $2 s-1$ on each side of the tube regardless of the direction and shape of the tube. $s$ is the scale of B-spline wavelet transform.

The proof of Lemma 3 is given in Appendix C. Obviously, Lemmas 2 and 3 are generally applicable for not only blood vessels but also any objects with tube-like geometrical structure. For illustration, an example is given to testify the correctness of these lemmas. Figure 2 shows the influence of local region size $d$ on the edge map estimated by using BWLSD from Fig. 1(f), which can be regarded as a crosssection of a 3-D tube. In Fig. 1, the chosen wavelet scale $s$ for Fig. 1(f) is 2 and therefore the proper value of $d$ should be 3 . Figure $1(\mathrm{~h})$ is the edge map obtained at $d=3$. If the value of $d$ is reduced to 2 , then the shrunken local region is unable to provide stable statistical estimations. As a consequence, see Fig. 2(b), some edge points are falsely classified into background since their local standard deviations become close to the average of that of background points

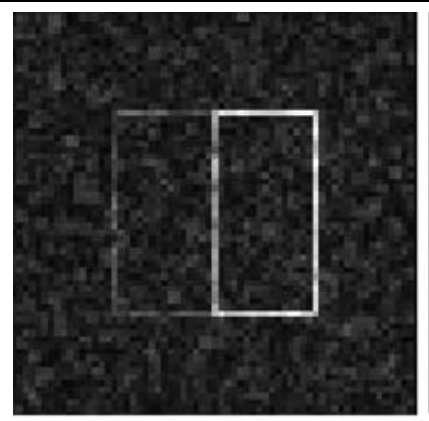

(a)

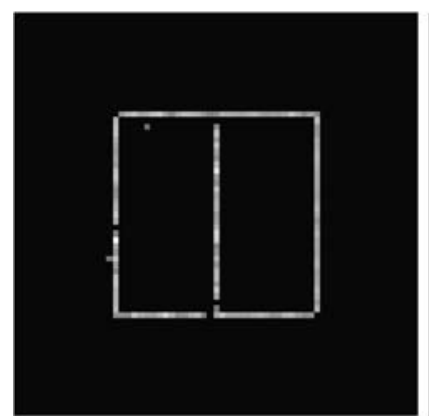

(c)

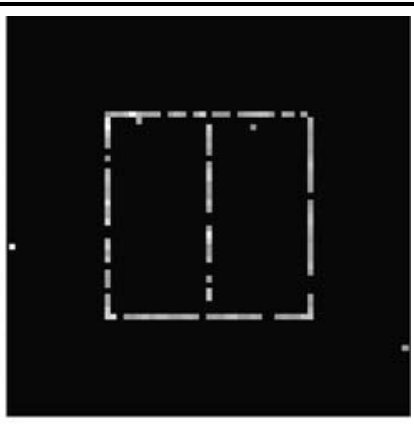

(b)

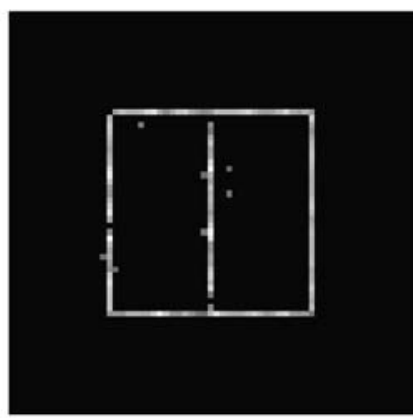

(d)
Fig. 2 This figure shows the edge maps obtained by applying BWLSD on the image shown in Fig. 1(f) with different values of local size $d$. (a) $d=1$. (b) $d=2$. (c) $d=5$. (d) $d=7$

and some background points are falsely classified into edges since their local standard deviations become close to the average of that of edge points. When the value of $d$ is reduced to 1 (see Fig. 2(a)), the local standard deviation of each point is zero. To prevent division by zero, we rewrite (12) as

$G(\mathbf{x})=\frac{\left|\nabla W_{s} f(\mathbf{x})\right|}{\sigma\left(\left|\nabla W_{s} f(R)\right|\right)+\epsilon}$,

where $\epsilon$ is a small non-zero constant and has the value of 0.01 in this paper. Obviously, when $d=1$, the local standard deviations of all points are the same and consequently the function BWLSD is reduced to the conventional waveletbased function. When the value of $d$ increases, some points in the smooth region, especially those near the edges, would be falsely classified as edge points since the enlarged local region leads to the increase of their local standard deviations. In addition, the number of falsely classified points augments along the increase of the value of $d$.

\subsection{FLUX for Image Segmentation}

After obtaining the reliable edge map by using BWLSD, we adopt FLUX (Vasilevskiy and Siddiqi 2002) to segment blood vessels in the angiographic images. The evolution of a closed active contour $C$ is given as

$C_{t}=\operatorname{div}(\vec{V}) \cdot \vec{N}$ 
where $t$ parameterizes the curve evolution family, $\vec{N}$ is an unit normal vector and $\vec{V}$ is a gradient vector field of an image $I$ smoothed by a Gaussian filter $G$, namely,

$\vec{V}=G * I$.

The zero level of a level set function (Osher and Sethian 1988 ) is used to characterize the evolving contour $C$. Accordingly, the evolution of the level set function $\psi$ is

$\psi_{t}=\alpha|\nabla \psi|+\beta\left(\nabla \cdot \frac{\nabla \psi}{|\nabla \psi|}\right)$,

where $\alpha$ is the speed function to control the evolving speed of contour $C$ along the normal direction and its value is $\operatorname{div}(\vec{V})$ in (16). Vasilevskiy and Siddiqi (2002) proposed a multi-scale method to calculate the value of $\operatorname{div}(\vec{V})$. $\left(\nabla \cdot \frac{\nabla \psi}{|\nabla \psi|}\right)$ is the mean curvature and $\beta$ is the regularization term to decide the contour smoothness. The sparse field set method (Whitaker 1998) is utilized to numerically solve (18) and set relevant parameters. We use the Insight Segmentation and Registration ToolKit (ITK) (Ibanez et al. 2005) to implement the level set method.

\section{Implementation Issues}

In Sect. 2, we have presented our method and the underlying design principles. In this section, we discuss the implementation issues of our method. The procedure of our method, the design of wavelet filters and setting of parameters will be given in this section.

\subsection{The Procedure of Our Method}

As a summary, the procedure of our proposed method for vascular segmentation is described as follows.

\section{The BWLSD-based edge detection method}

1. Rescaling the voxel size in the original image volume so that the voxel dimensions are isotropic.

2. Selecting a proper scale of wavelet transform $s$ according to the properties of the image volume, such as the minimum radius of blood vessels and noise level.

3. Designing the wavelet filters based on the B-spline smooth, wavelet functions and the selected wavelet scale $s$.

4. Calculating the wavelet magnitudes $\left|\nabla W_{s} f(\mathbf{x})\right|$ and corresponding direction vectors $(\alpha, \beta, \gamma)$ by convolving the input image with the designed wavelet filters.

5. Calculating the local standard deviation of the image volume. According to the Lemma 2 proposed in Sect. 2.3 the size of local region is $2 s-1$ when the wavelet scale is $s$.
6. Setting the wavelet magnitude of smooth region to zero by global thresholding based on local standard deviation. That is to say, the wavelet magnitude of such a voxel whose local standard deviation is smaller than the threshold value is set to zero.

7. Normalizing the wavelet magnitude by dividing corresponding local standard deviation. The normalized wavelet magnitude is used as the edge strength.

8. Applying the FLUX to segment the blood vessels based on the obtained edge map.

A short explanation of this algorithm procedure is given in the following. The physical sizes along $\mathrm{x}, \mathrm{y}$, and $\mathrm{z}$ axes of one voxel in the original image volume are not same, as a consequence, this leads to distortion. To annihilate this distortion, a rescaling operation to make those sizes equal is necessary. A proper wavelet scale $s$ is critical for our algorithm to obtain a good segmentation result. A large scale $s$ is capable of removing the noise interference at the expense of deducing the accuracy of edge detection. On the contrary, a small scale can accurately detect the edge of thin vessels but at the same time is sensitive to the noise. In discrete image processing, the wavelet transform is practically implemented by convolution operation. According to the separability of B-spline wavelet function, the 3-D B-spline wavelet transform can be turned into 1-D B-spline wavelet transform. Consequently, the design of 3-D wavelet filters is replaced by the design of 1-D wavelet filters, as it greatly reduces the complexity of our method. We will discuss the design of B-spline wavelet filters at length in the next subsection. Regarding Step 6, in spite of the existence of intensity inhomogeneity, the local standard deviation of smooth intensity region (corresponding to background region) is distinctive from that of sharply transiting intensity region (corresponding to edge region). By selecting a proper threshold value, the thresholding (Step 6) of local variations can remove most of the background voxels. The remaining background voxels, if any, are isolated. In addition, in implementing Step 7, one voxel's wavelet magnitude is normalized by dividing the maximum value of the local standard deviations of itself and its neighbors instead of its local standard deviation. In this way, the measured edge strength of the background voxels around an edge region is much lower than that of the edge voxels. Therefore, via these two steps, the remaining background voxels cannot attract the FLUX active contour. As such, leakage to background can be avoided. After the normalization in Step 7, the edge strengths in regions with different intensity contrasts are the same and thus the effect of intensity inhomogeneity can be minimized. 
3.2 Discrete Implementation of 3-D B-spline Wavelet Transform

So far, the discussion on B-spline wavelet transform is restricted in the continuous domain. In practice, the discrete wavelet transform is very important since images in the real world are in discrete form. In this subsection, we will discuss the discrete wavelet function and the approach to calculate the corresponding wavelet coefficients.

Since multi-dimensional B-spline smooth function and wavelet function are separable, we get:

$\left\{\begin{array}{l}\theta_{s}(\mathbf{x})=\theta_{s}(x) \theta_{s}(y) \theta_{s}(z), \\ \psi_{s}(\mathbf{x})=\psi_{s}(x) \psi_{s}(y) \psi_{s}(z) .\end{array}\right.$

Thus, the multi-dimensional B-spline wavelet functions can be rewritten as

$\left\{\begin{array}{l}\psi_{s}^{1}(\mathbf{x})=\psi_{s}(x) \theta_{s}(y) \theta_{s}(z), \\ \psi_{s}^{2}(\mathbf{x})=\theta_{s}(x) \psi_{s}(y) \theta_{s}(z), \\ \psi_{s}^{3}(\mathbf{x})=\theta_{s}(x) \theta_{s}(y) \psi_{s}(z) .\end{array}\right.$

The wavelet transforms in (6) can be rewritten as

$\left\{\begin{array}{l}W_{s}^{1} f(\mathbf{x})=f(\mathbf{x}) * \psi_{s}(x) * \theta_{s}(y) * \theta_{s}(z), \\ W_{s}^{2} f(\mathbf{x})=f(\mathbf{x}) * \theta_{s}(x) * \psi_{s}(y) * \theta_{s}(z), \\ W_{s}^{3} f(\mathbf{x})=f(\mathbf{x}) * \theta_{s}(x) * \theta_{s}(y) * \psi_{s}(z) .\end{array}\right.$

It is clear that the 3-D wavelet transforms expressed in (6) are 3-D convolutions, of which the calculation cost is very huge. By utilizing (21), the 3-D wavelet transforms can be turned from 3-D convolutions into 1-D convolutions and thus the calculation cost can be greatly reduced. As such, to some extent, (21) can be regarded as the fast calculation version of (6). 1-D B-spline smooth function $\theta_{s}(x)$ and 1-D B-spline wavelet function $\psi_{s}(x)$ are graphically displayed in Figs. 3(a) and 3(b), respectively. The wavelet scale $s$ plays a very important role on controlling image denoising and the location accuracy of edge detection. The larger the value of $s$ is, the more smooth the wavelet function is while the weaker localization ability the wavelet function has. In practice, the ideal edges are optimally detected by considering the trade-off in both localization and smoothness (Canny 1986; Mallat and Hwang 1992).

The smooth function $\theta$ and the wavelet function $\psi$ can be viewed as the impulse responses of a low-pass filter $G$ and a high-pass filter $H$, respectively. Thereby, the corresponding wavelet transforms are able to be implemented by convolving the image $f(\mathbf{x})$ with filters $G$ and $H$ in certain orders. That is, in a 3-D square integrable space $L^{2}\left(R^{3}\right)$, the discrete wavelet transform is described below:

$\left\{\begin{array}{l}W_{s}^{1} f(\mathbf{x})=f(\mathbf{x}) * H_{s} * G_{s} * G_{s}, \\ W_{s}^{2} f(\mathbf{x})=f(\mathbf{x}) * G_{s} * H_{s} * G_{s}, \\ W_{s}^{3} f(\mathbf{x})=f(\mathbf{x}) * G_{s} * G_{s} * H_{s} .\end{array}\right.$

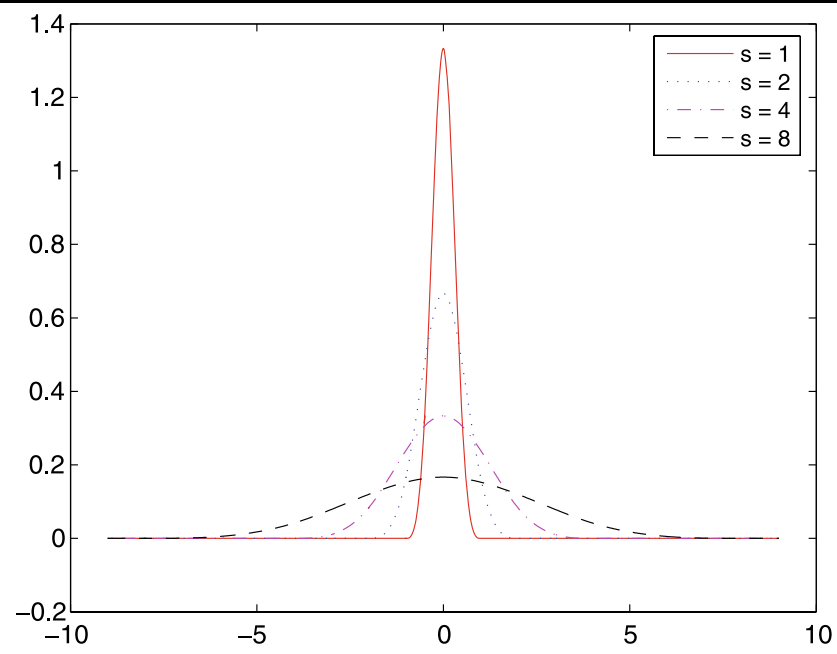

(a)

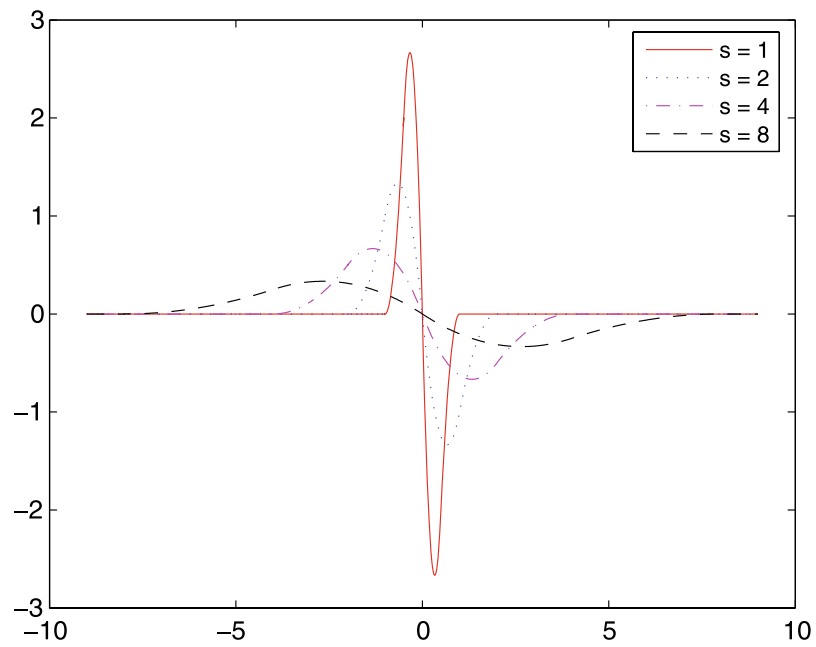

(b)

Fig. 3 (a) The 1-D B-spline smooth functions at different scales. (b) The 1-D B-spline wavelet functions at different scales

From (21) and (22), the 1-D convolution along $\mathrm{x}$ axis is

$$
\left\{\begin{aligned}
f(\mathbf{x}) * \theta_{s}(x) & =\int f(u, y, z) \theta_{s}(x-u) d u \\
& =\sum_{k} f(k, y, z) \int_{k}^{k+1} \theta_{s}(x-u) d u \\
& =f(\mathbf{x}) * G_{s}(x), \\
f(\mathbf{x}) * \psi_{s}(x) & =\int f(u, y, z) \psi_{s}(x-u) d u \\
& =\sum_{k} f(k, y, z) \int_{k}^{k+1} \psi_{s}(x-u) d u \\
& =f(\mathbf{x}) * H_{s}(x) .
\end{aligned}\right.
$$

The 1-D convolutions along $\mathrm{y}$ and $\mathrm{z}$ axes are similar.

So, if we define the sequences $\left\{g_{k}\right\},\left\{h_{k}\right\}$ as the coefficients of $G_{s}$ and $H_{s}$, respectively, then we have

$\left\{\begin{array}{l}g_{s, k}=\int_{k}^{k+1} \theta_{s}(x) d x=\int_{\frac{k}{s}}^{\frac{k+1}{s}} \theta(x) d x, \\ h_{s, k}=\int_{k}^{k+1} \psi_{s}(x) d x=\int_{\frac{k}{s}}^{\frac{k+1}{s}} \psi(x) d x .\end{array}\right.$ 
Table 1 High-pass filter coefficients $\left\{h_{s, k}\right\}$

\begin{tabular}{rcccr}
\hline$K$ & $S=1$ & $S=2$ & $S=4$ & $S=8$ \\
\hline-8 & $/$ & $/$ & $/$ & 0.0064 \\
-7 & $/$ & $/$ & $/$ & 0.0452 \\
-6 & $/$ & $/$ & $/$ & 0.1225 \\
-5 & $/$ & $/$ & $/$ & 0.2384 \\
-4 & $/$ & $/$ & 0.0375 & 0.3674 \\
-3 & $/$ & $/$ & 0.2623 & 0.4060 \\
-2 & $/$ & 0.2236 & 0.5622 & 0.3286 \\
-1 & 0.7071 & 0.6708 & 0.3373 & 0.1354 \\
0 & -0.7071 & -0.6708 & -0.3373 & -0.1354 \\
1 & $/$ & -0.2236 & -0.5622 & -0.3286 \\
2 & $/$ & $/$ & -0.2623 & -0.4060 \\
3 & $/$ & $/$ & -0.0375 & -0.3674 \\
4 & $/$ & $/$ & $/$ & -0.2384 \\
5 & $/$ & $/$ & $/$ & -0.1225 \\
6 & $/$ & $/$ & $/$ & -0.0452 \\
7 & $/$ & $/$ & $/$ & -0.0064 \\
\hline
\end{tabular}

Table 2 Low-pass filter coefficients $\left\{g_{s, k}\right\}$

\begin{tabular}{rllll}
\hline$K$ & $S=1$ & $S=2$ & $S=4$ & $S=8$ \\
\hline-8 & $/$ & $/$ & $/$ & 0.0004 \\
-7 & $/$ & $/$ & $/$ & 0.0007 \\
-6 & $/$ & $/$ & $/$ & 0.0307 \\
-5 & $/$ & $/$ & $/$ & 0.0827 \\
-4 & $/$ & $/$ & 0.0054 & 0.1723 \\
-3 & $/$ & $/$ & 0.0810 & 0.2883 \\
-2 & $/$ & 0.0064 & 0.3292 & 0.3988 \\
-1 & 0.7071 & 0.7042 & 0.6205 & 0.4695 \\
0 & 0.7071 & 0.7042 & 0.6205 & 0.4695 \\
1 & $/$ & 0.0064 & 0.3292 & 0.3988 \\
2 & $/$ & $/$ & 0.0810 & 0.2883 \\
3 & $/$ & $/$ & 0.0054 & 0.1723 \\
4 & $/$ & $/$ & $/$ & 0.0827 \\
5 & $/$ & $/$ & $/$ & 0.0307 \\
6 & $/$ & $/$ & $/$ & 0.0007 \\
7 & $/$ & & & 0.0004 \\
\hline
\end{tabular}

These two sequences can be further normalized into

$$
\left\{\begin{array}{l}
\sum_{k} g_{s, k}^{2} \approx 1, \\
\sum_{k} h_{s, k}^{2} \approx 1 .
\end{array}\right.
$$

We calculate all filter coefficients $\left\{g_{s, k}\right\}$ and $\left\{h_{s, k}\right\}$ numerically and list them in Tables 1 and 2.
3.3 Setting Parameters of the Proposed Method

As for the parameters of FLUX, we closely follow the configuration described in Law and Chung (2007). Two criteria are utilized to find the initial seeds. One criterion is that seeds must have intensity values larger than the presetting threshold. The other criterion is that all neighbors around selected seeds within radius $r$ also have intensity values larger than the presetting threshold ( $r=1$ in this paper). The second step is capable of removing the disturbance caused by isolated noise. The presetting threshold value $T$, in practice, is calculated via the following formula,

$T=\operatorname{prctile}(I, p)$,

where $I$ is the input image, $p$ is a percentage, prctile is a function which returns an intensity value according to the intensity distribution of image $I$ and the percentage $p$. In other words, $T$ is such an intensity value that is larger than the intensity of $p \%$ voxel of image $I$. The value of smoothness regularization term $\beta$, as stated in (18), is set empirically. A large value of $\beta$ makes the FLUX evolution surface become very smooth while a small value of $\beta$ may stop the evolution surface at an unexpected position. In other words, although more vessels can be segmented by FLUX with a smaller value of $\beta$, the smoothness of the vessel surface simultaneously reduces and the possibility of occurrence of leakages simultaneously increases. Therefore, the value of $\beta$ should be set carefully. In our work, we empirically set $\beta=0.05$.

\section{Experimental Results}

In this section, we first validate our proposed edge detection method, BWLSD, on synthetic images ranging from 2-D images to 3-D images, from test objects with corners (viz. rectangles) to objects without corners (viz. circle), from images with intensity inhomogeneity alone to images with both intensity inhomogeneity and noise. These tests are designed to demonstrate that BWLSD is an effective edge detection framework for images under different conditions, e.g. intensity inhomogeneity and noise. FLUX embedded with BWLSD, namely BWLSD-FLUX, is then applied to synthetic images containing intensity inhomogeneity as well as white Gaussian noise, and six clinical MRA image volumes including three PC-MRA image volumes and three TOF-MRA image volumes to evaluate its performance on segmentation. As will be shown in this section, the promising segmentation results of clinical experiments reveal that BWLSD-FLUX is able to achieve quality segmentation of vasculatures in MRA images. 
Fig. 4 Comparisons on a 2-D image with intensity inhomogeneity but without noise. (a) The original 2-D image containing several rectangles with same length but different widths. (b) The edges detected by using GRADIENT. (c) The edges detected by using BWLSD

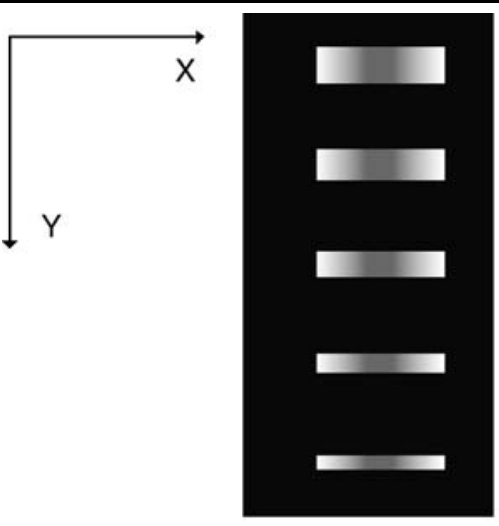

(a)

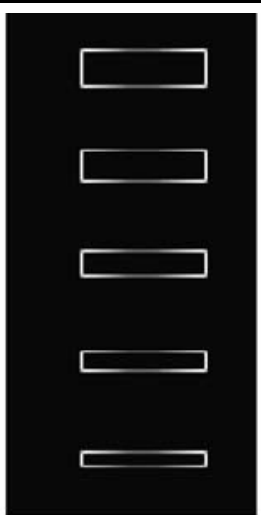

(b)

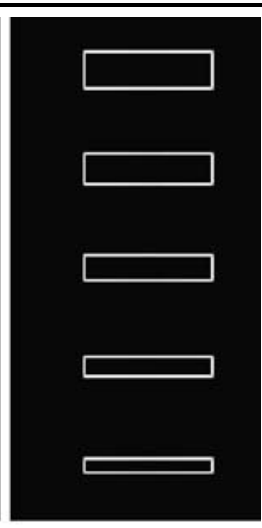

(c)

\subsection{Synthetic Images}

In this subsection, BWLSD is tested on different types of 2-D and 3-D synthetic images and its performances on edge detection are demonstrated and analyzed. First, a 2-D synthetic image of size $180 \times 100$ pixels, as shown in Fig. 4(a), is used for experiments. This image contains five bright rectangles with same length but different widths. The widths, from top to bottom, are 13, 11, 9, 7, and 5 pixels, respectively. These rectangles can be regarded as 2-D cross sections of 3-D tubes with different radii. The intensity values of pixels inside these rectangles are consistent along the $y$ axis while varying along the $\mathrm{x}$-axis. The intensity $I(x, y)$ along the $\mathrm{x}$-axis varies and is defined as

$I(x, y)= \begin{cases}1-(x-31) \times 0.025, & x \in[31,50] \& y \in \tilde{Y}, \\ 0.5, & x \in[51,60] \& y \in \tilde{Y}, \\ 1-(80-x) \times 0.025, & x \in[61,80] \& y \in \tilde{Y}, \\ 0, & \text { otherwise. }\end{cases}$

Here, the set $\tilde{Y}$ refers to a collection of y coordinates inside the tubes. The intensity range of this 2 -D image is $[0,1]$, and the background intensity is zero. In this section, BWLSD is compared with the gradient-based method. Hereinafter, the gradient-based method is referred to GRADIENT for easy reference. For BWLSD, the edge strength is estimated according to (12). Edge strength estimated by using GRADIENT is computed according to the following equation:

$M=\sqrt{\left(I * G_{x}\right)^{2}+\left(I * G_{y}\right)^{2}+\left(I * G_{z}\right)^{2}}$,

where $G_{x}, G_{y}$ and $G_{z}$ represent the first derivatives of a Gaussian function along $\mathrm{x}, \mathrm{y}$ and $\mathrm{z}$ axes, respectively. The edges detected by using GRADIENT and BWLSD are shown in Figs. 4(b) and 4(c), respectively.

For Fig. 4, the intensity profiles along the top horizontal boundaries of the rectangles with different widths are plotted in Fig. 5. Without loss of generality, Fig. 5 only considers the $x$ coordinates within $[31,80]$, where the rectangles lie. Figure 5(a) displays the intensity profiles of five rectangles from top to bottom. From this figure, it can be observed that the intensity fluctuates radically in the original image along the $x$-axis. Figure 5(b) indicates that GRADIENT cannot deal with the problem of intensity inhomogeneity and the intensity variation profile of GRADIENT nearly duplicates the intensity profile of original image. However, as plotted in Fig. 5(c), the intensity variation profiles of BWLSD with $s=2$ are very close to a straight line although there are two slight turning points at $x=50$ and $x=60$. These slight turning points are caused by two singularities of intensity changing function, as stated in (27), at $x=50$ and $x=60$, where the intensity changes sharply. In addition, to make a quantitative comparison, we measure the standard deviations of all the intensity profiles along the horizontal boundaries of the rectangles in the original image (Fig. 4(a)), GRADIENT edge map (Fig. 4(b)) and BWLSD edge map (Fig. 4(c)). Note that there are 10 horizontal intensity profiles for each image/map. The average standard deviations of the horizontal intensity profiles of the original image, GRADIENT edge map and BWLSD edge map are $0.1644,0.1640$ and 0.0232 , respectively. It is revealed that, for BWLSD, even with the effect of intensity inhomogeneity, the estimated edge strengths are relatively consistent along the rectangle boundaries and independent of the widths of rectangles.

Next, we consider a 2-D image with intensity inhomogeneity as well as noise. Such an image is created by introducing random noise into the image used in the previous experiment (see Fig. 4(a)). That is,

$I 1(x, y)=I(x, y)+0.03 * \operatorname{randn}()$,

where $I 1$ is the image with intensity inhomogeneity and noise, $I$ is the original input image, as shown in Fig. 4(a), 0.03 is the standard deviation of the white Gaussian function, $\operatorname{randn}(\cdot)$ is a random function to generate normally distributed random numbers with zero mean and $\sigma=1$. The image with the white Gaussian noise is shown in Fig. 6(a), in 

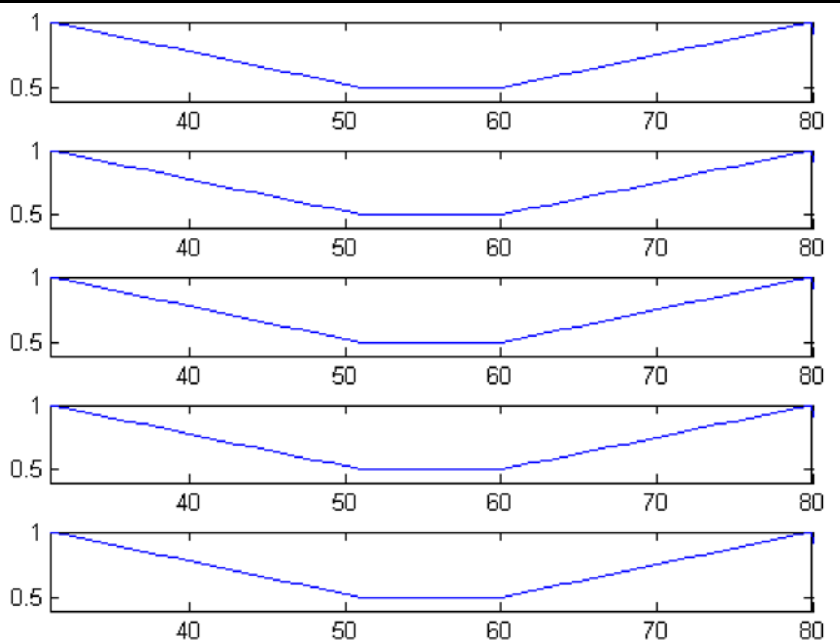

(a)
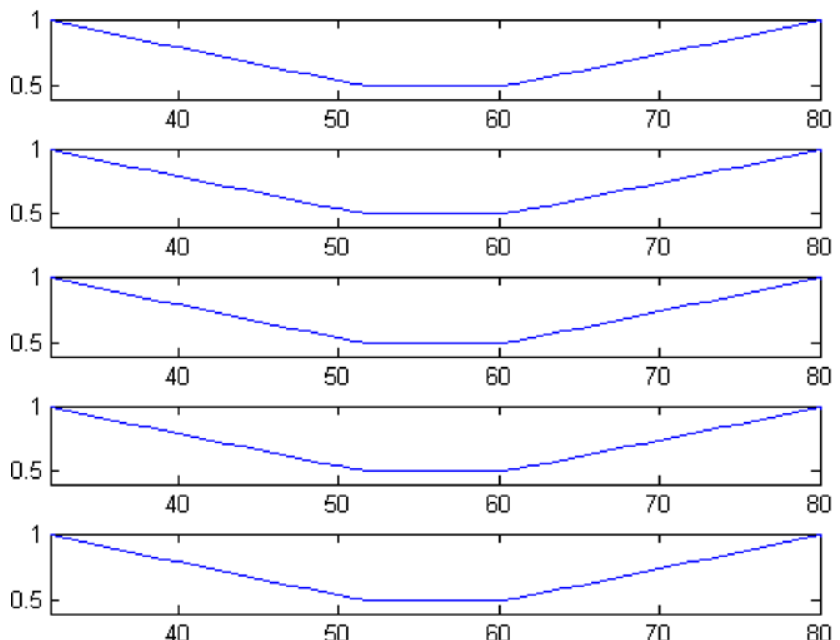

(b)
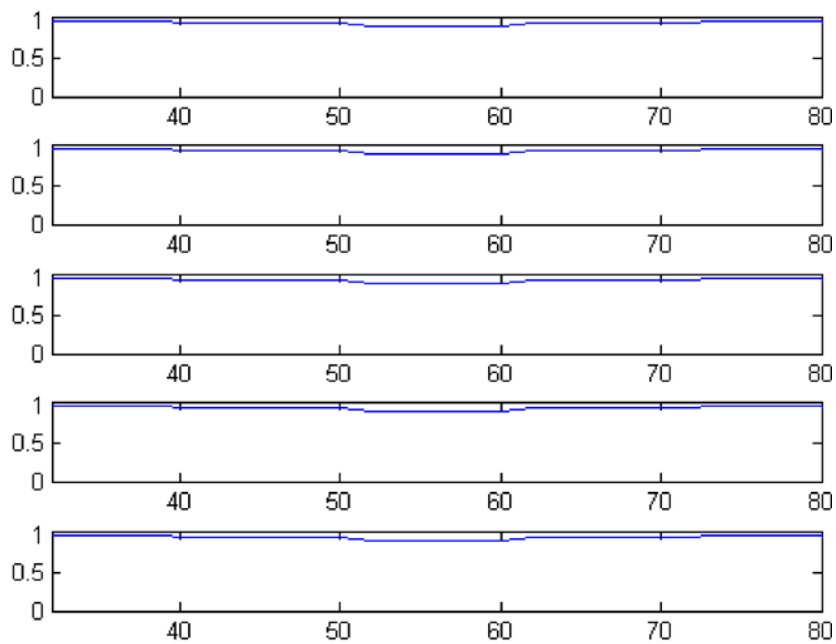

(c)

Fig. 5 Intensity profiles along the top horizontal boundaries of rectangles with different widths. (a) Original image. (b) GRADIENT. (c) BWLSD

which both GRADIENT and BWLSD are tested. The edges are detected by using GRADIENT and BWLSD with $s=2$ according to (28) and (12), respectively. The detected edges are shown in Figs. 6(b) and 6(c). From Fig. 6(c), as compared with Fig. 6(b), it is observed that BWLSD not only gives consistent edge strengths under intensity inhomogeneity but also reduces the noise level in the image.

The intensity inhomogeneity caused by bias field, a deficiency of MRA acquisition systems, is one major and inherent problem for MRA image segmentation and can lead to serious misclassification for intensity-based segmentation techniques. Therefore, a basic requirement for one robust MRA segmentation technique is the ability to correct the bias field in the MR images. In the next set of experiments, we illustrate the ability of BWLSD against varying bias fields. The bias fields (bias fields $A, B, C$ ) we used are obtained from BrainWeb (http://www.bic.mni.mcgill.ca//

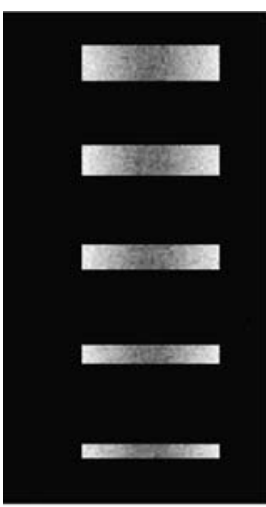

(a)

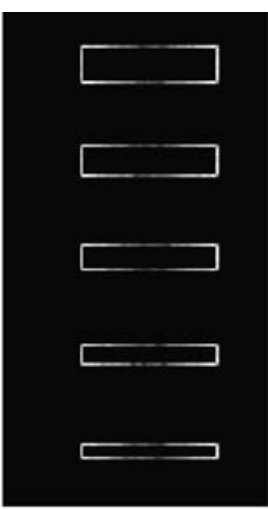

(b)

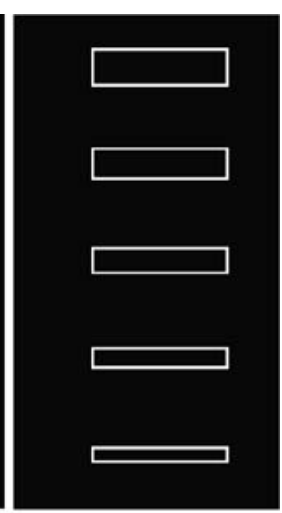

(c)
Fig. 6 Comparisons on a 2-D image with both intensity inhomogeneity and white Gaussian noise. (a) The original 2-D image. (b) The edges detected by using GRADIENT. (c) The edges detected by using BWLSD 


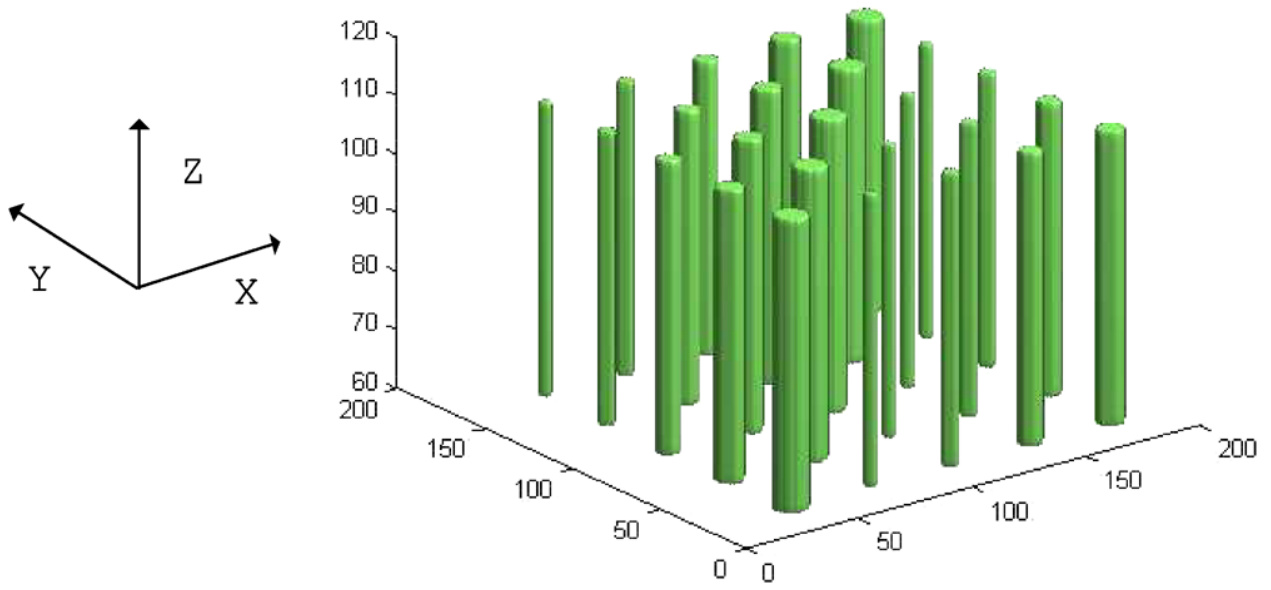

Fig. 7 A 3-D image containing 25 tubes with same lengths but different radii

/brainweb/), a public simulated brain database. These bias fields are realistic since they were estimated from real MRA scans.

A 3-D image is created for testing. It consists of 25 tubes with different radii ranging from 2 to 10 pixels, as shown in Fig. 7. In fact, the previously used 2-D image can be regarded as a slice of this 3-D image along y-axis. In this experiment set, these bias fields and white Gaussian noise with $\sigma=0.03$ are added to the 3-D image. This model can be described by the following equation:

$I 1(x, y)=I(x, y) * F(x, y)+0.03 * \operatorname{randn}()$,

where $I 1$ is the $3-\mathrm{D}$ image after being added the bias field and noise, $I$ is the original 3-D image and $F$ is the applied bias field. In this experiment, $F$ is selected from bias fields $A, B$ and $C$. All of these three bias fields have $20 \%$ intensity non-uniformity (INU). $20 \%$ level means the multiplicative INU field, which can be linearly scaled, has a range of values of $0.90, \ldots, 1.10$ over the brain image. For simplicity, we denote the 3-D image affected by noise with $\sigma=0.03$ and bias field $\mathrm{A}$ as $I_{A}$, by bias field $\mathrm{B}$ as $I_{B}$, and by bias field $\mathrm{C}$ as $I_{C}$.

Figure 8(a) shows a slice of the original image $I$ in the $\mathrm{x}-\mathrm{z}$ plane at $y=35$. At the corresponding locations, Figs. 8(b), 8(c) and 8(d) are the image slices of $I_{A}, I_{B}$ and $I_{C}$, respectively. The edges detected by using GRADIENT and BWLSD with $s=2$ are shown in Figs. 8(e)-(g), and Figs. 8(h)-(j). In order to further illustrate the performances of GRADIENT and BWLSD, Figs. 9(a), 9(b) and 9(c) show the slices in the $\mathrm{x}-\mathrm{y}$ plane at $z=85$ of $I_{A}, I_{B}$ and $I_{C}$, respectively. Figures $9(\mathrm{~d})-(\mathrm{f})$ are the edges detected by using GRADIENT, and Figs. 9(g)-(i) are their edges detected by using BWLSD. From these figures, we can see that BWLSD is not adversely affected by the intensity inhomogeneity caused by the bias fields and white Gaussian noise.

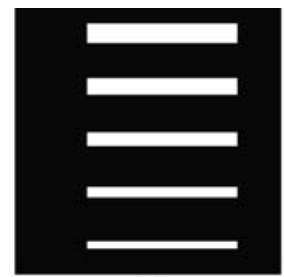

(a)

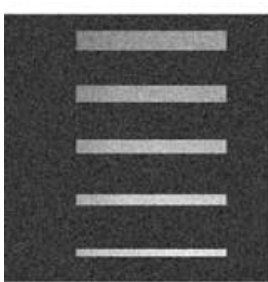

(b)

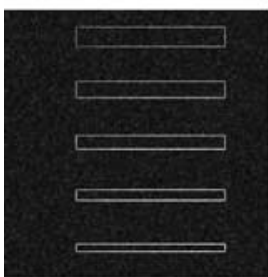

(e)

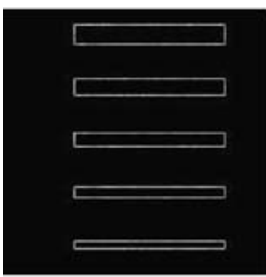

(h)

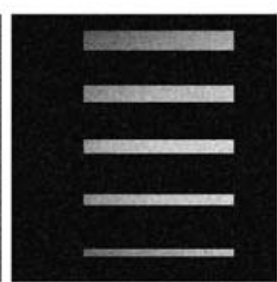

(c)

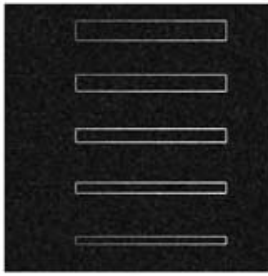

(f)

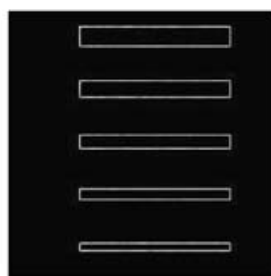

(i)

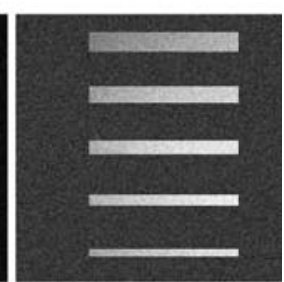

(d)

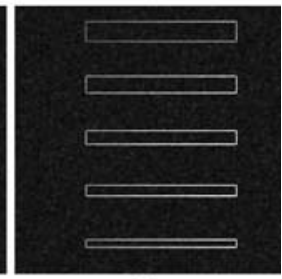

(g)

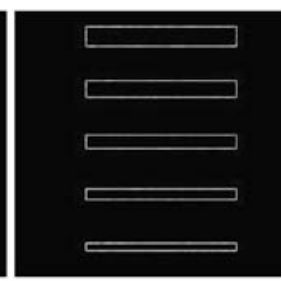

(j)
Fig. 8 An illustrating example at one slice in the $x-z$ plane. (a) One slice of the original 3-D image I at $y=35$. (b, c, d) are the slices of $I_{A}, I_{B}$ and $I_{C}$, respectively, also at $y=35$. $(\mathbf{e}, \mathbf{f}, \mathbf{g})$ and $(\mathbf{h}, \mathbf{i}, \mathbf{j})$ are the edges of $(b, c, d)$ detected by using GRADIENT and BWLSD, respectively 


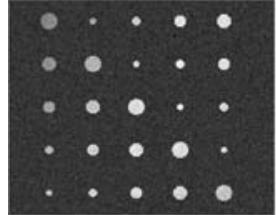

(a)

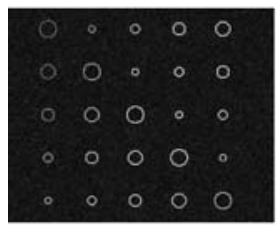

(d)

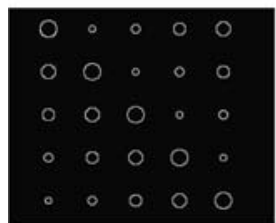

(g)

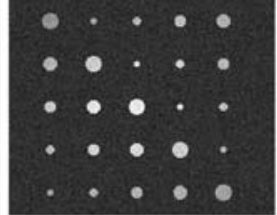

(b)

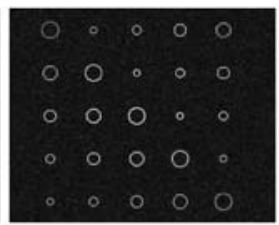

(e)

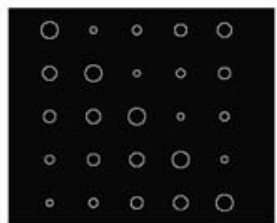

(h)

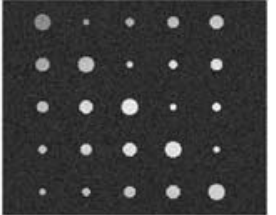

(c)

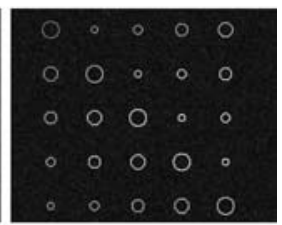

(f)

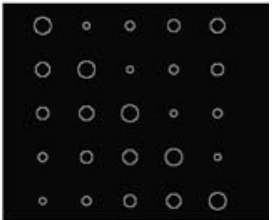

(i)
Fig. 9 Another illustrating example at one slice in the $x-y$ plane. $(\mathbf{a}, \mathbf{b}, \mathbf{c})$ are slices at $z=85$ of $I_{A}, I_{B}$ and $I_{C}$, respectively. (d, e, f) and $(\mathbf{g}, \mathbf{h}, \mathbf{i})$ are edges of $(\mathrm{a}, \mathrm{b}, \mathrm{c})$ detected by using GRADIENT and BWLSD, respectively
Statistical distributions of edge strengths are employed in order to quantify the performance of GRADIENT and BWLSD. Figures 10(a), 10(b) and 10(c) are the statistical distributions of the edge strengths of all edge voxels detected by using GRADIENT in $I_{A}, I_{B}$ and $I_{C}$, respectively. As a comparison, the statistical distributions of the edge strengths of all edge voxels of $I_{A}, I_{B}, I_{C}$ corrected by BWLSD are shown in Figs. 10(d), 10(e) and 10(f). Obviously, the intensity distributions of edges corrected by BWLSD are more concentrating in a narrower range than GRADIENT. This phenomenon implies that the strength values of edge voxels disturbed by the bias field are successfully narrowed down into a comparatively shorter range. Therefore, the intensity values of edge voxels, no matter where it locates in the 3-D image, are less fluctuating. As such, it is suggested that the intensity inhomogeneity caused by bias fields are well smoothed out. In addition, we also measure the standard deviations of these statistical intensity distributions to have a quantitative comparison. The standard deviations of Figs. 10(a), 10(b), 10(c), 10(d), 10(e) and 10(f) are 0.1686, $0.1603,0.1633,0.1175,0.1176$ and 0.1186 , respectively. These numerical results also indicate that BWLSD, as compared with GRADIENT, are more robust to intensity inhomogeneity and noise.

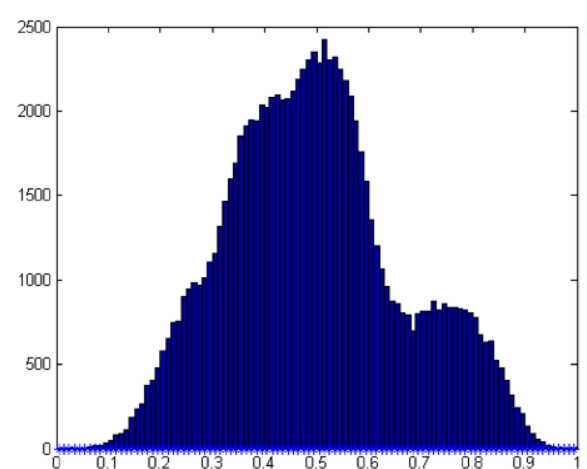

(a)

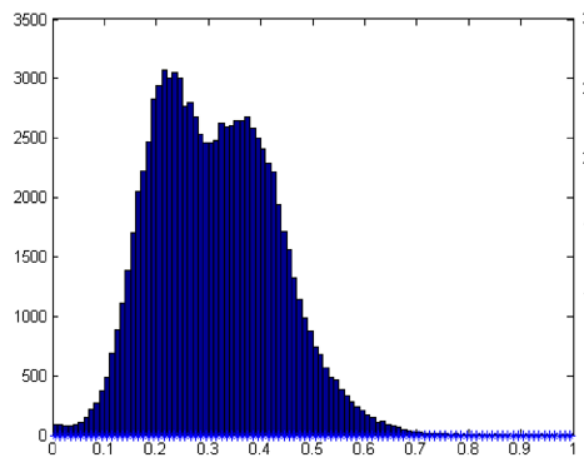

(d)

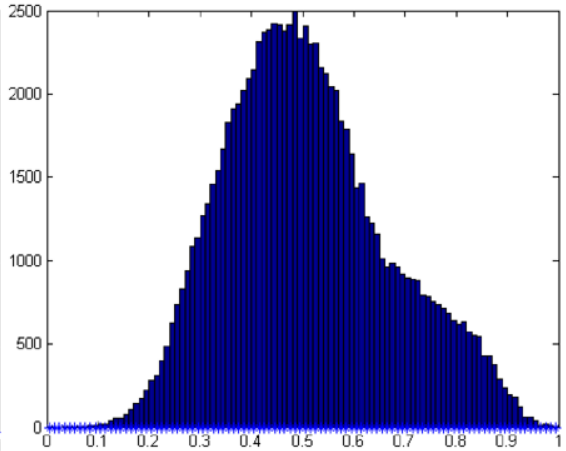

(b)

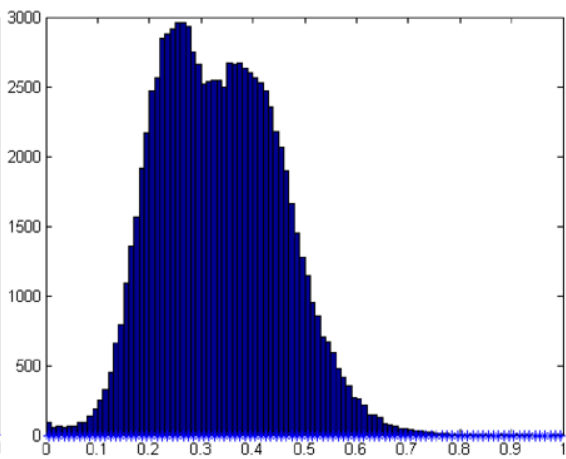

(e)

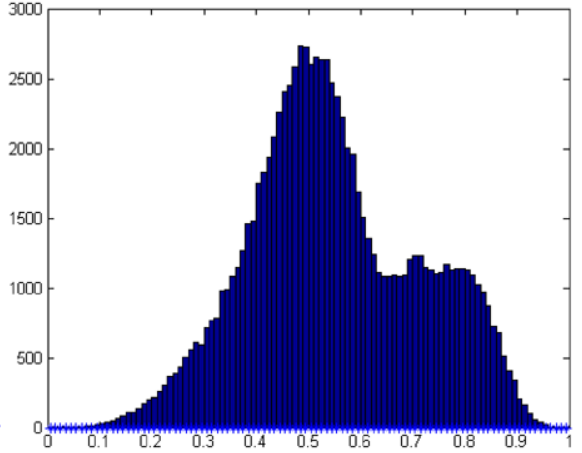

(c)

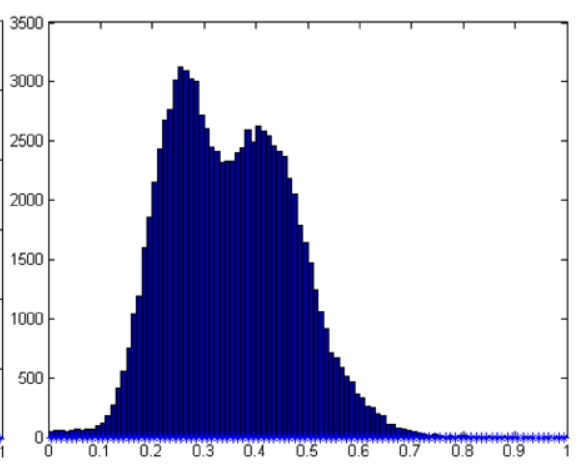

(f)

Fig. 10 Statistical distributions of edge strength. $(\mathbf{a}, \mathbf{b}, \mathbf{c})$ are distributions of edge strength obtained by using GRADIENT. (d, e, f) are distributions of edge strength obtained by using BWLSD 
Fig. 11 Performances on a 2-D image composed by three nested hollow circles. (a) The original image. (b) One hollow circle is determined by two parameters: $R$ and $r$. (c) The image contaminated by intensity inhomogeneity alone. $(\mathbf{d}, \mathbf{e})$ are edges of (c) detected by using GRADIENT and BWLSD, respectively. (f) The image contaminated by both intensity inhomogeneity and white Gaussian noise. $(\mathbf{g}, \mathbf{h})$ are edges of (f) detected by using GRADIENT and BWLSD, respectively

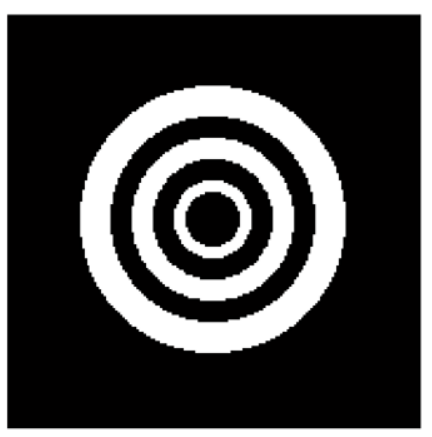

(a)

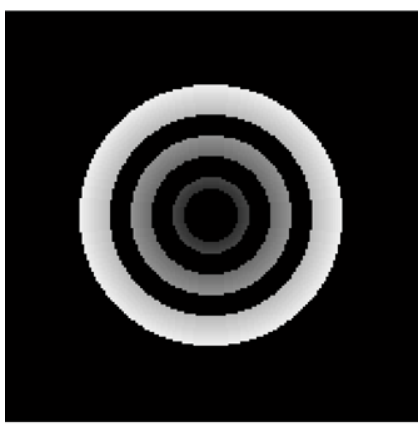

(c)

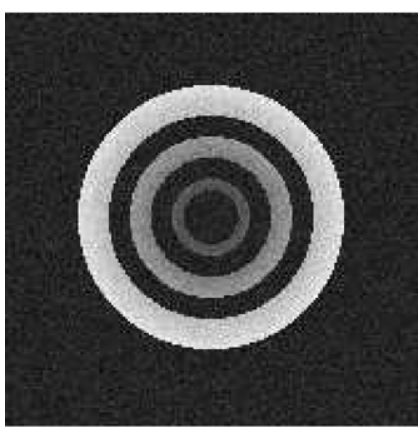

(f)

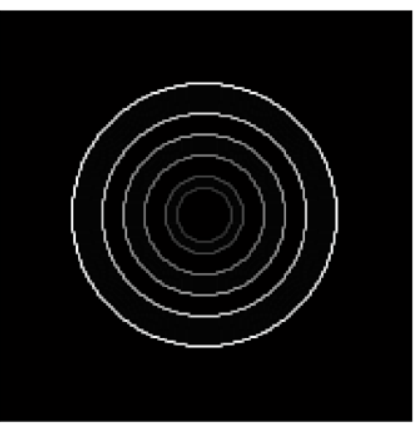

(d)

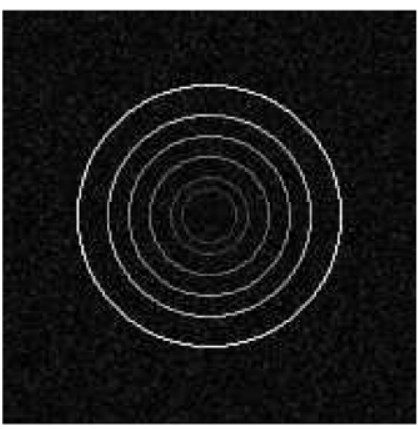

(g)

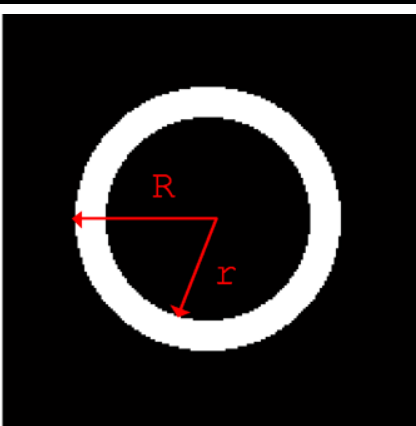

(b)

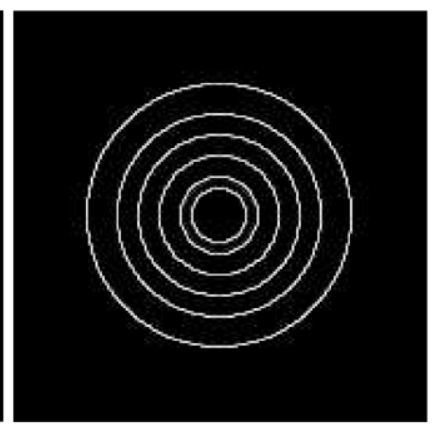

(e)

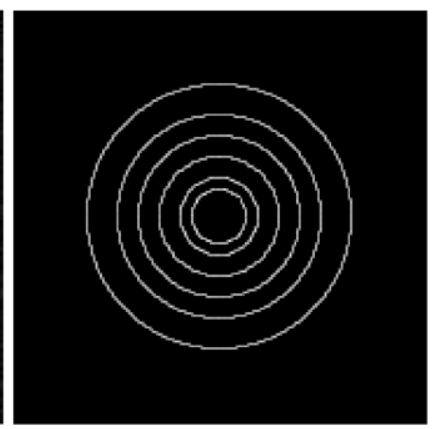

(h)
In the previous experiments, we compare BWLSD with GRADIENT on 2-D images, in which the rectangular objects are composed based on straight lines. We now compare these two methods on image, in which the objects are composed based on curves. One representative of such objects is hollow circle. We denote one hollow circle as $\{R, r\}$ since a hollow circle can be represented by two parameters: $R$ (the radius of outer circle) and $r$ (the radius of inner circle), as shown in Fig. 11(b). Figure 11(a) shows three nested hollow bright circles: $\{56,44\},\{34,26\}$ and $\{16,12\}$ in a dark background. We create intensity inhomogeneity by assigning one pixel's intensity inversely proportional to the distance between this pixel and the central point, as shown in Fig. 11(c). The edges of Fig. 11(c) detected by using GRADIENT are shown in Fig. 11(d). The figure illustrates that GRADIENT can accurately detect the edges but still sensitive to the intensity inhomogeneity because the edge strengths vary between the outer ring and inner ring. On the contrary, BWLSD is capable of accurately detecting edges as well as smoothing out the effect of intensity inhomogeneity simultaneously, as demonstrated in Fig. 11(e). Similar to the previous experiments, in this experiment, we also tested GRADIENT and BWLSD on the image contaminated by both intensity inhomogeneity and white Gaussian noise, as shown in Fig. 11(f). The edges detected by using GRADIENT and BWLSD with $s=2$ are displayed in Figs. 11(g) and 11(h), respectively. It is very clear that the noise and intensity inhomogeneity do not adversely affect the quality of the edge map generated by using BWLSD while, in contrast, still affect the quality of the edge map generated by using GRADIENT.

Torus is a natural 3-D extension of hollow circle. We create a 3-D image consisting of 12 tori with varied parameters. The tori can be divided into 4 groups. From top to bottom, from outside to inside, using the convention $\{R, r\}$, these tori are denoted as $\{46,34\},\{24,16\},\{72,48\},\{36,24\}$, $\{14,10\},\{48,32\},\{24,16\},\{46,34\},\{56,44\},\{34,26\}$ and 
Fig. 12 A 3-D image containing 12 tori with different parameters

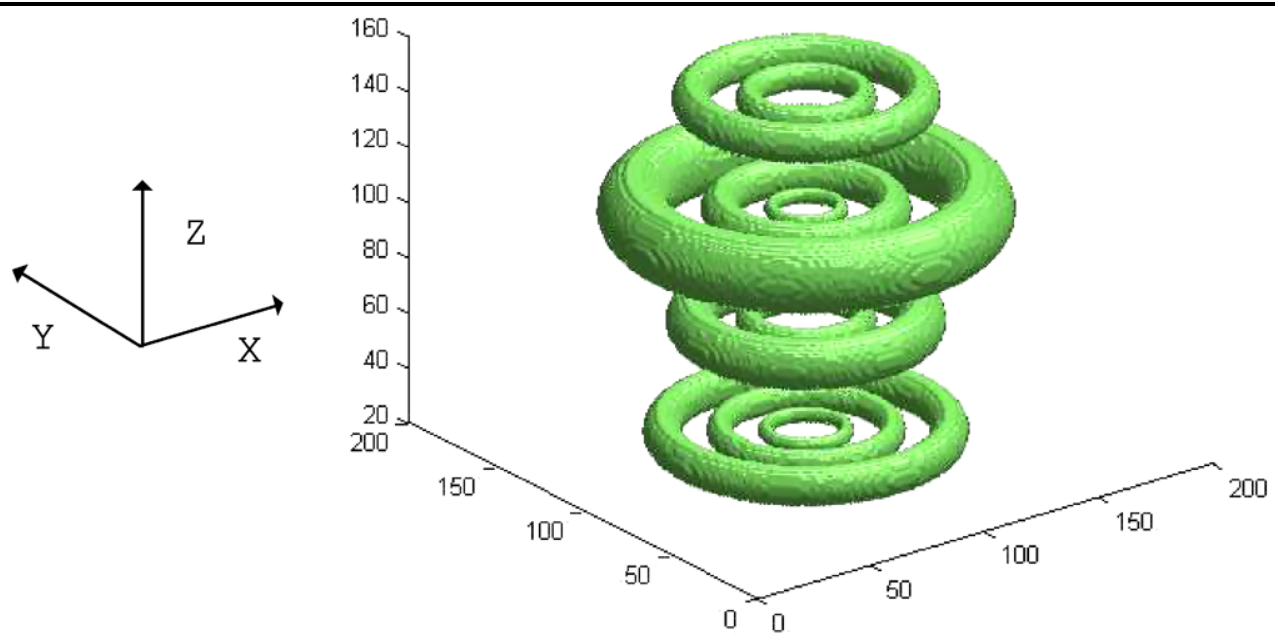

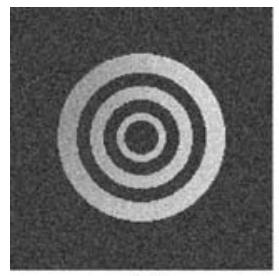

(a)

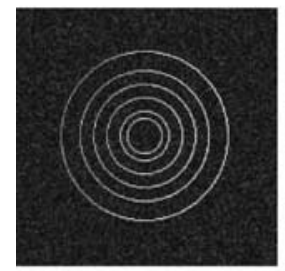

(d)

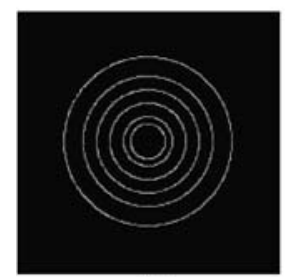

(g)

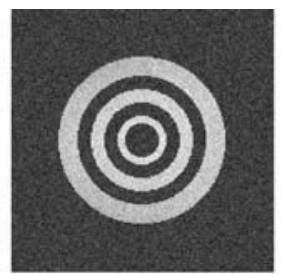

(b)

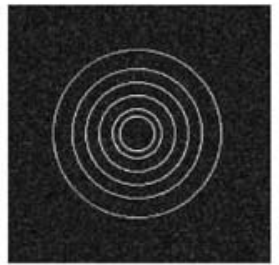

(e)

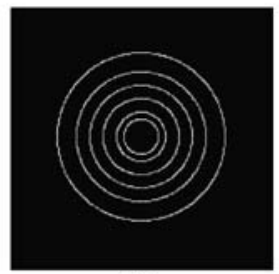

(h)

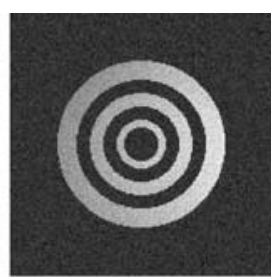

(c)

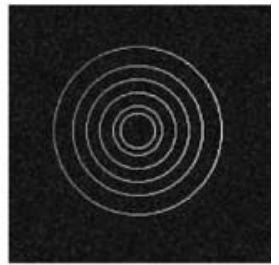

(f)

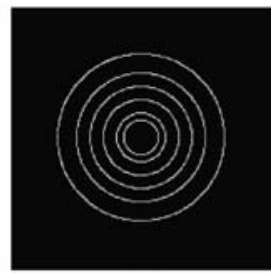

(i)
Fig. 13 An illustrating example using one slice at $z=30$. (a, b, c) are slices at $z=30$ of $I_{A}, I_{B}$ and $I_{C}$, respectively. $(\mathbf{d}, \mathbf{e}, \mathbf{f})$ and $(\mathbf{g}, \mathbf{h}$, i) are edges of $(a, b, c)$ detected by using GRADIENT and BWLSD, respectively

$\{16,12\}$, as shown in Fig. 12. Figure 13(a) is just one slice of this image at $z=30$. Similarity, the bias fields $A, B, C$ and white Gaussian noise with $\sigma=0.03$ are applied to this image and the corresponding contaminated images are denoted as $I_{A}, I_{B}$ and $I_{C}$. Using the slices at $z=150$, a direct illustrative example is given in Fig. 13. Figures 13(a), 13(b) and 13(c) are the corresponding slices of $I_{A}, I_{B}$ and $I_{C}$, respectively. Figures $13(\mathrm{e})-(\mathrm{g})$ are edges detected by using GRADIENT and Figs. 13(h)-(j) are edges detected by using
BWLSD with $s=2$. It is clear that the edges detected by using BWLSD are more resistant to intensity inhomogeneity and noise. Similar to the previous experiments, Figs. 14(a), 14(b) and 14(c) show the statistical distributions of edge strengths of all selected edge voxels obtained by using GRADIENT from $I_{A}, I_{B}$ and $I_{C}$, of which the standard deviations are 0.1892, 0.1949, 0.1894, respectively. For BWLSD, Figs. 14(d), 14(e) and 14(f) show the statistical distributions of edge strengths and the standard deviations are 0.1340 , $0.1153,0.1410$, respectively. All of these experiments illustrate that BWLSD is a reliable edge detection technique for images with intensity inhomogeneity and noise, and independent of the geometrical structure of image objects.

In the next set of experiments, we use two kinds of synthetic images to test the segmentation ability of BWLSDFLUX. The first synthetic image for testing is a tube with radius $r=5$ voxels and length $l=50$ voxels, as shown in Fig. 15. The second image for testing is a torus $\{22,14\}$, as shown in Fig. 18. These images are binary images in which the intensity of background voxels is zero and the intensity of tube or torus voxels is one. To illustrate the ability of BWLSD-FLUX against intensity inhomogeneity and noise, these images are contaminated by different kinds of bias field (bias fields $A, B$ and $C$ ) and different levels of white Gaussian noise with $\sigma=0.01,0.03,0.05$, respectively. The scale of the B-spline wavelet filters used here is 2 . As the examples to display the quality of contaminated images, Figs. 16 and 19 show the middle slices of the contaminated tube and torus images, respectively.

Dice similarity coefficient (Zijdenbos et al. 1994; Gooya et al. 2008) is adopted here to measure the segmentation errors. The segmentation errors of BWLSD-FLUX and FLUX on contaminated tube and torus images are listed in Tables 3 and 4, respectively. Obviously, BWLSD-FLUX has a better performance than FLUX because BWLSD can provide a better edge map for FLUX, as illustrated in the above ex- 


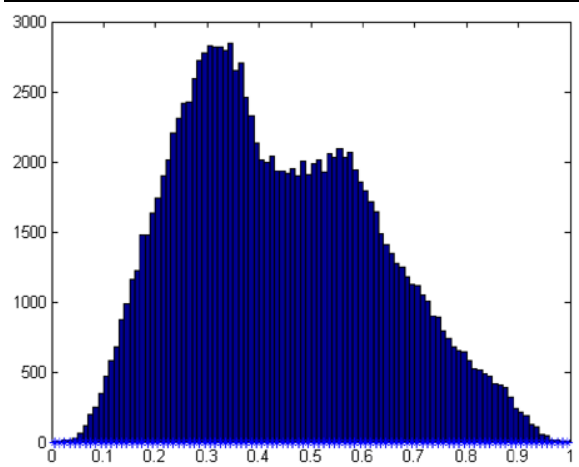

(a)

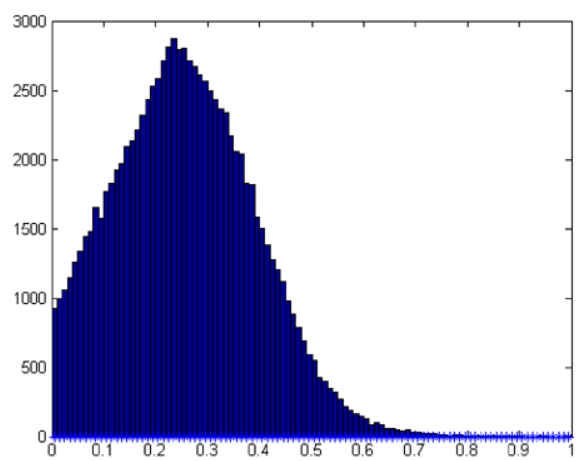

(d)

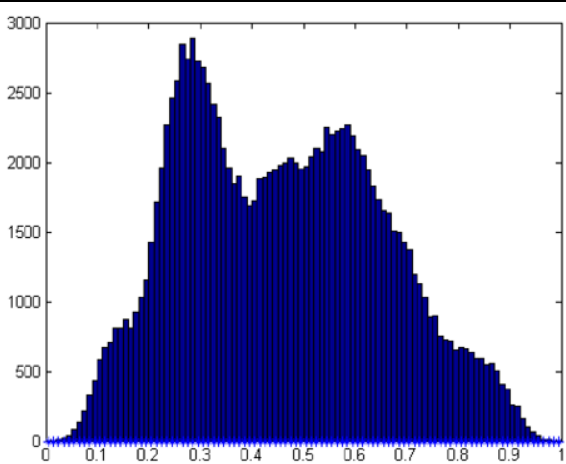

(b)

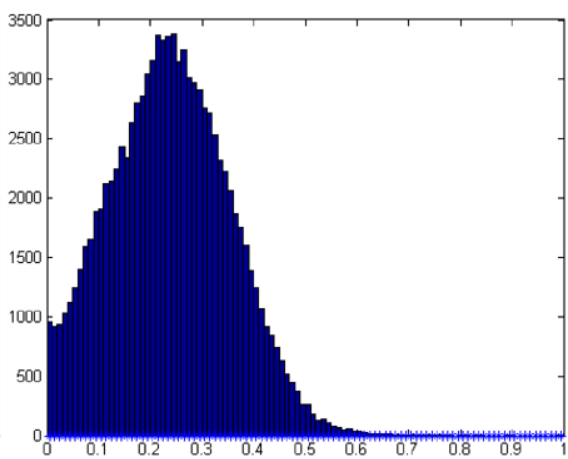

(e)

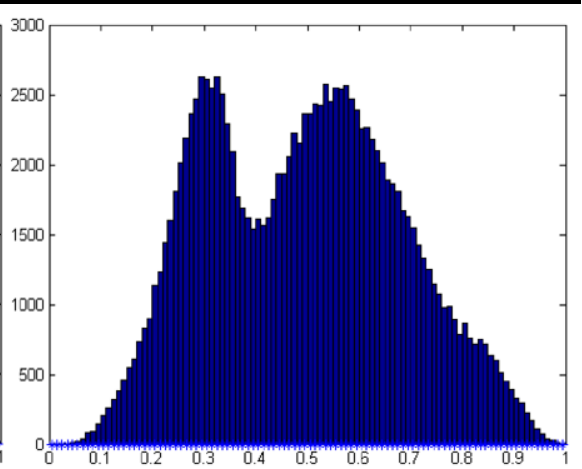

(c)

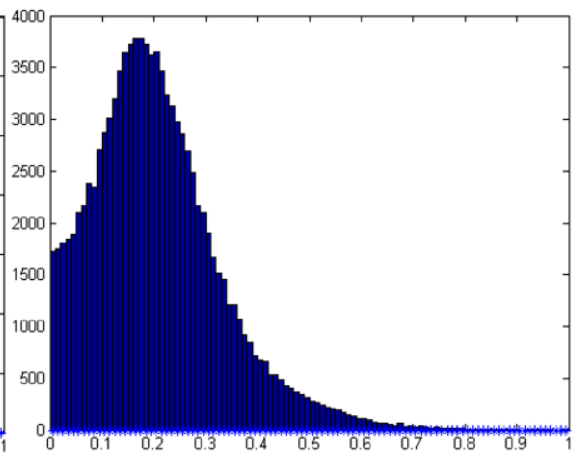

(f)

Fig. 14 Statistical distributions of edge strength. $(\mathbf{a}, \mathbf{b}, \mathbf{c})$ are distributions of edge strength obtained by using GRADIENT. (d, e, f) are distributions of edge strength obtained by using BWLSD

Fig. 15 A testing image with a tube inside, radius $r=5$ voxels and length $l=50$ voxels

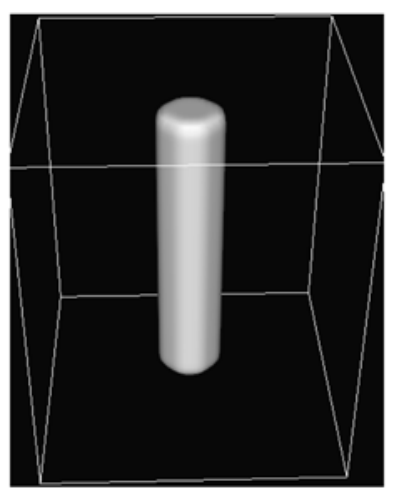

periments. It should be noted that there is no significant difference between the segmentation results of BWLSD-FLUX and that of FLUX on these synthetic images. The reason is that the contrast variation introduced by the bias fields $A, B$ and $C$ are not large. While the contrast variation becomes much larger in clinical MRA images, it is observed that some low contrast vessels can be segmented by BWLSDFLUX while cannot by FLUX.

\subsection{Clinical Images}

In the previous subsection, we have tested BWLSD-FLUX's ability against intensity inhomogeneity and noise on syn-

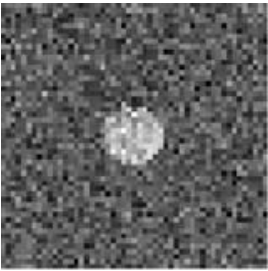

(a)

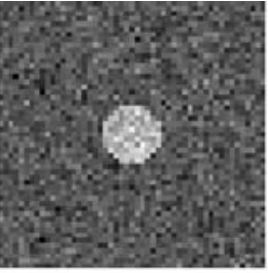

(b)

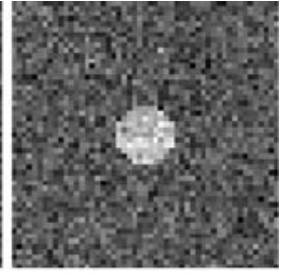

(c)
Fig. 16 The middle slices of the tube images contaminated by white Gaussian noise $\sigma=0.05$ and different bias fields. (a) Bias field $A$. (b) Bias field $B$. (c) Bias field $C$

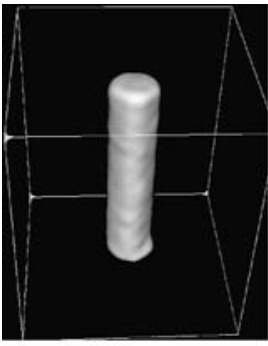

(a)

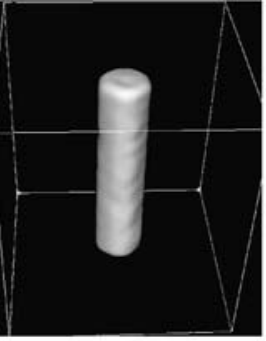

(b)

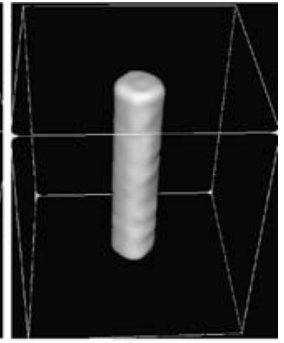

(c)
Fig. 17 BWLSD-FLUX segmentation results of the tube images contaminated by white Gaussian noise $\sigma=0.05$ and different bias fields, respectively. (a) Bias field $A$. (b) Bias field $B$. (c) Bias field $C$ 
thetic images. In this subsection, we test BWLSD-FLUX for vascular segmentation in 3-D clinical MRA images and compare BWLSD-FLUX with FLUX. These MRA images were scanned by a Philips 3T ACS Gyroscaln MR scanner

Fig. 18 The tested torus image

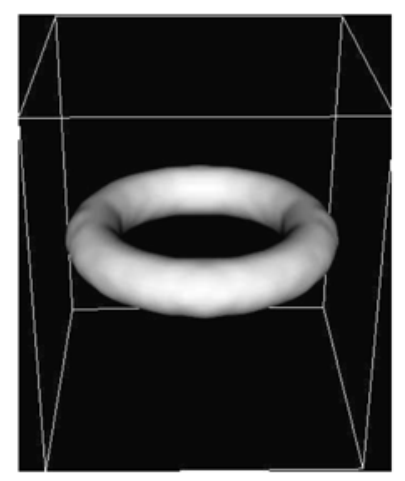

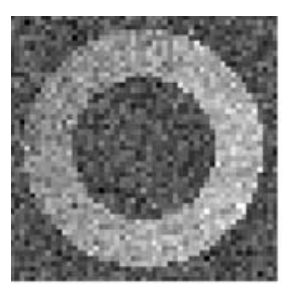

(a)

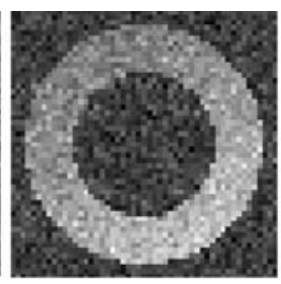

(b)

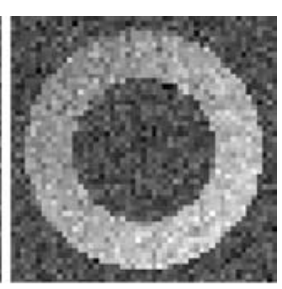

(c)
Fig. 19 The middle slice of the torus images contaminated by white Gaussian noise $\sigma=0.05$ and different bias fields. (a) Bias field $A$. (b) Bias field $B$. (c) Bias field $C$

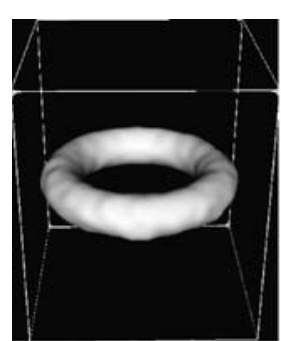

(a)

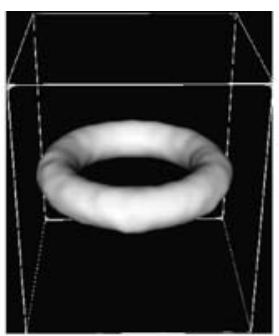

(b)

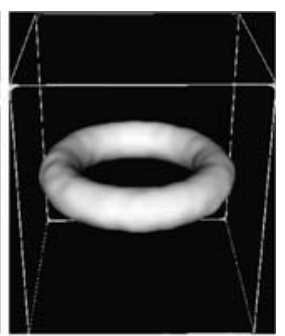

(c)
Fig. 20 BWLSD-FLUX segmentation results of the torus images contaminated by white Gaussian noise $\sigma=0.05$ and different bias fields, respectively. (a) Bias field A. (b) Bias field $B$. (c) Bias field $C$ at the University Hospital of Zurich, Switzerland. The image sizes and voxel dimensions are listed in Table 5. In this table, $X, Y, Z$ refer to the numbers of voxels of each image volume along $\mathrm{x}, \mathrm{y}, \mathrm{z}$ axes, respectively, and $x, y, z$ mean voxel dimensions along $\mathrm{x}, \mathrm{y}, \mathrm{z}$ axes respectively.

Figure 21 illustrates and compares the performances of BWLSD-FLUX $(s=2)$ and FLUX on a $104 \times 252 \times 64$ PC-MRA image volume, which is named Image 1 in this paper. Maximum intensity projections (MIPs) of this PC-MRA image volume are shown in Fig. 21(a) and the first row of Fig. 22. The initial seed image is shown in Fig. 21(b), in which the seeds are found by setting $p=99.9 \%$ in (26). The segmentation result of FLUX is shown in Fig. 21(c). The contour halts at several low contrast boundaries labeled by the red dotted circles from 1 to 8 in the MIP image and as a result misses a large portion of the brain vasculature. These 8 labeled boundaries do not represent all low con-

Table 3 Segmentation errors of FLUX and BWLSD-FLUX on a tube image contaminated by white Gaussian noise and bias field

\begin{tabular}{lllll}
\hline Method & Bias field & $\sigma=0.01$ & $\sigma=0.03$ & $\sigma=0.05$ \\
\hline BWLSD-FLUX & $A$ & $11.45 \%$ & $13.30 \%$ & $14.41 \%$ \\
& $B$ & $11.24 \%$ & $12.46 \%$ & $13.45 \%$ \\
& $C$ & $11.37 \%$ & $12.54 \%$ & $13.71 \%$ \\
\hline FLUX & $A$ & $12.05 \%$ & $16.07 \%$ & $18.16 \%$ \\
& $B$ & $11.74 \%$ & $14.74 \%$ & $17.49 \%$ \\
& $C$ & $11.87 \%$ & $15.23 \%$ & $17.85 \%$ \\
\hline
\end{tabular}

Table 4 Segmentation errors of FLUX and BWLSD-FLUX on a torus image contaminated by white Gaussian noise and bias field

\begin{tabular}{lllll}
\hline Method & Bias field & $\sigma=0.01$ & $\sigma=0.03$ & $\sigma=0.05$ \\
\hline BWLSD-FLUX & $A$ & $12.98 \%$ & $13.28 \%$ & $13.77 \%$ \\
& $B$ & $12.43 \%$ & $12.94 \%$ & $13.42 \%$ \\
& $C$ & $12.56 \%$ & $13.14 \%$ & $13.60 \%$ \\
\hline FLUX & $A$ & $13.64 \%$ & $14.27 \%$ & $14.51 \%$ \\
& $B$ & $13.20 \%$ & $13.80 \%$ & $14.16 \%$ \\
& $C$ & $13.52 \%$ & $13.95 \%$ & $14.32 \%$ \\
\hline
\end{tabular}

Table 5 Image sizes and voxel dimensions of MRA images used in the experiments

\begin{tabular}{llllllll}
\hline Name & $\begin{array}{l}\text { Scanning } \\
\text { sequences }\end{array}$ & $X$ & $Y$ & $Z$ & $x$ & $y$ & $z$ \\
\hline Image1 & PC-MRA & 104 voxels & 252 voxels & 64 voxels & $0.40 \mathrm{~mm}$ & $0.40 \mathrm{~mm}$ & $1.00 \mathrm{~mm}$ \\
Image2 & PC-MRA & 130 voxels & 286 voxels & 52 voxels & $0.40 \mathrm{~mm}$ & $0.40 \mathrm{~mm}$ & $1.00 \mathrm{~mm}$ \\
Image3 & TOF-MRA & 239 voxels & 209 voxels & 60 voxels & $0.41 \mathrm{~mm}$ & $0.41 \mathrm{~mm}$ & $0.95 \mathrm{~mm}$ \\
Image4 & PC-MRA & 120 voxels & 256 voxels & 58 voxels & $0.39 \mathrm{~mm}$ & $0.39 \mathrm{~mm}$ & $0.90 \mathrm{~mm}$ \\
Image5 & TOF-MRA & 115 voxels & 256 voxels & 60 voxels & $0.39 \mathrm{~mm}$ & $0.39 \mathrm{~mm}$ & $0.95 \mathrm{~mm}$ \\
Image6 & TOF-MRA & 110 voxels & 260 voxels & 64 voxels & $0.39 \mathrm{~mm}$ & $0.39 \mathrm{~mm}$ & $0.85 \mathrm{~mm}$ \\
\hline
\end{tabular}


Fig. 21 An illustration of BWLSD-FLUX on

segmentation of a $104 \times 252 \times 64$ brain PC-MRA image. (a) The maximum intensity projection (MIP) of PC-MRA image. (b) The seed image. (c) The segmentation result obtained by using FLUX. (d) An evolution step of BWLSD-FLUX, of which the evolution front has just passed the low contrast boundaries labeled as 5, 6, 8. (d) An evolution step of

BWLSD-FLUX, of which the evolution front has passed the low contrast boundaries 1, 3, 4, 5, 6, 7, 8. (e) An evolution step of BWLSD-FLUX, of which the evolution front has passed the low contrast boundaries 1, 2, 3, 4, 5, 6, 7, 8. (g) The final segmentation result obtained by using BWLSD-FLUX

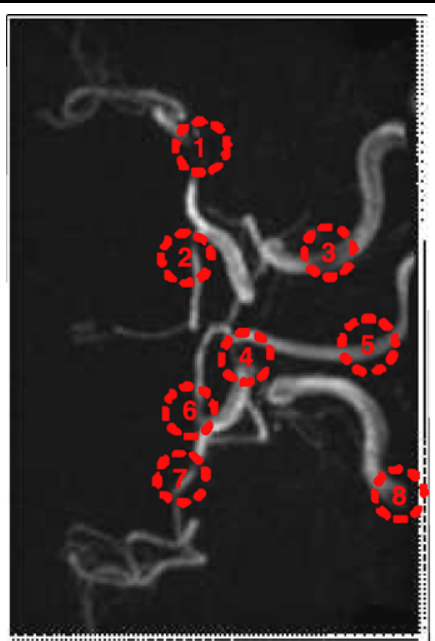

(a)

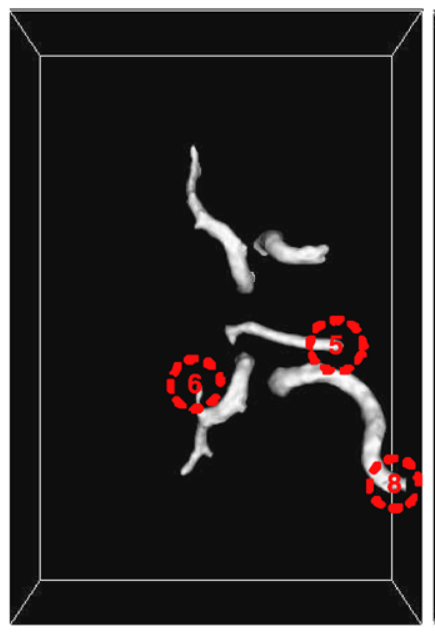

(d)

(g)

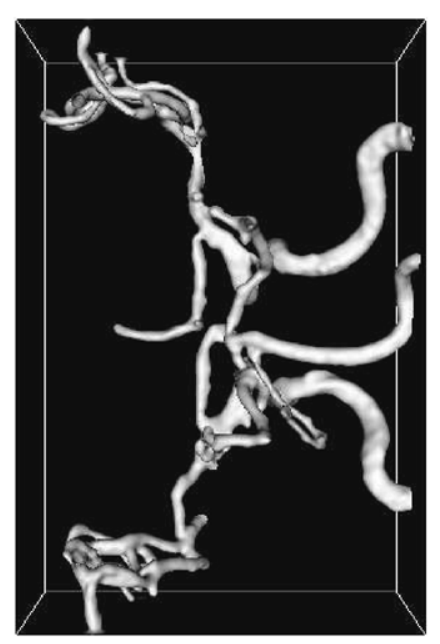

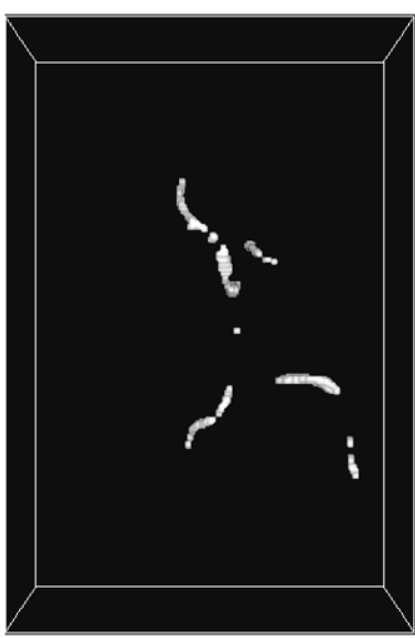

(b)

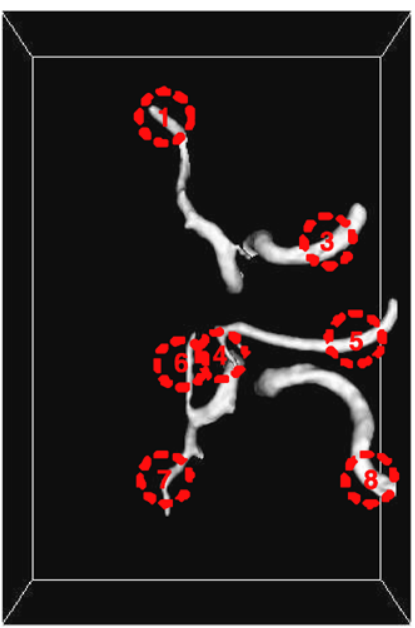

(e)

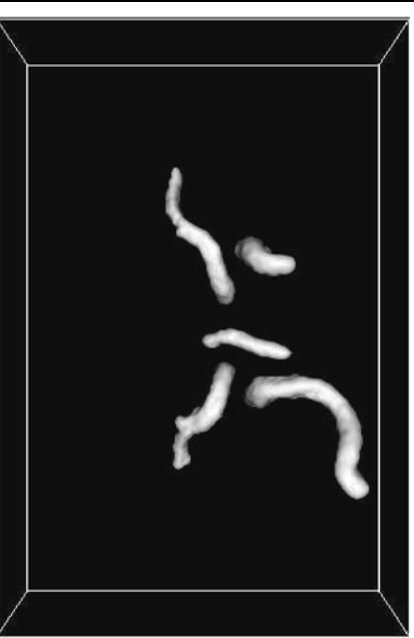

(c)

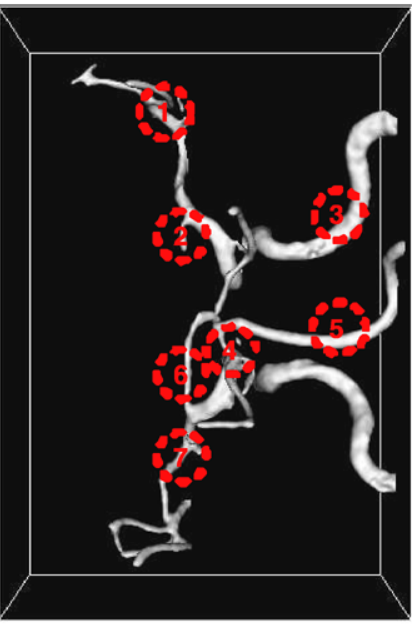

(f) trast boundaries in this image but only indicate the boundaries where FLUX wrongly halts when it starts from the initial seeds. Figures 21(d)-(f) are several evolution steps of
BWLSD-FLUX. These results demonstrate how BWLSDFLUX propagates through the low contrast boundaries. Figure $21(\mathrm{~g})$ is the final segmentation result of BWLSD-FLUX. 
Fig. 22 Two distinct views of maximum intensity projection (the first row) and the segmentation result obtained by using BWLSD-FLUX (the second row)
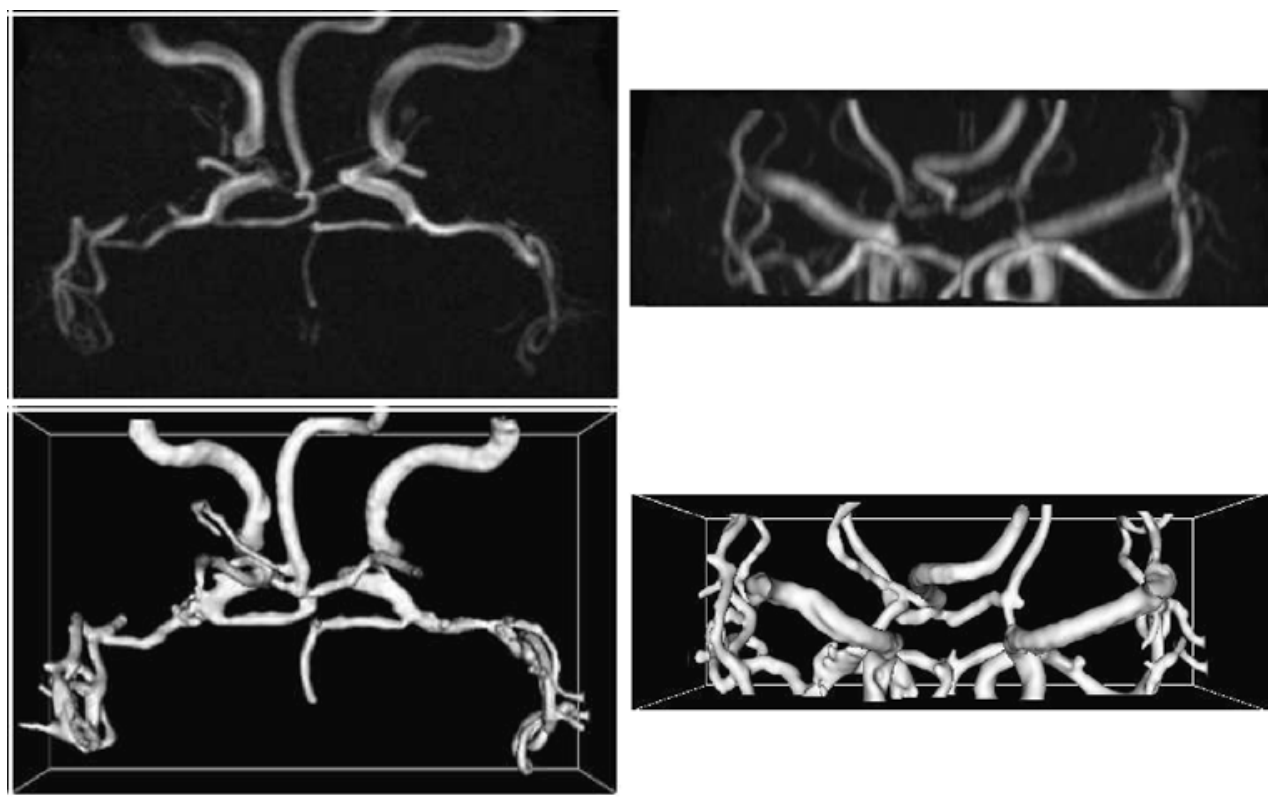

Fig. 23 Two distinct views of seeds of $p=99.5 \%$ (the first row) and the segmentation result obtained by using FLUX started from these seeds (the second row)
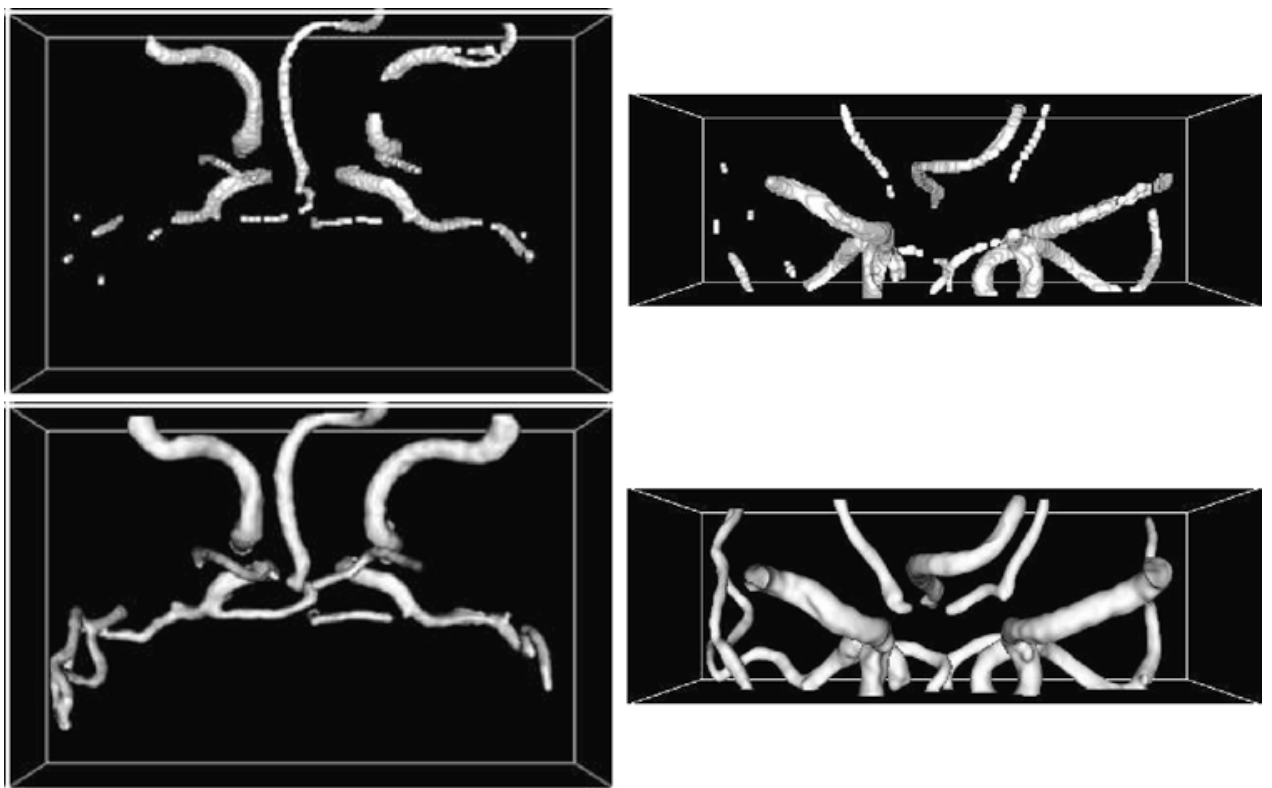

Apparently, BWLSD-FLUX successfully segments all vessels from the PC-MRA image and the segmented vasculature does not have any incorrect discontinuity. To comprehensively show the effectiveness of BWLSD-FLUX, the two distinct views of the MIP and the segmentation result of BWLSD-FLUX are shown in Fig. 22. Regarding the regularization parameters $\beta$, we have mentioned in Sect. 3.3, its value is selected as 0.05 .

This experiment testing BWLSD-FLUX and FLUX is based on a tough initial condition, which helps to exhibit the ability of BWLSD-FLUX to solve the problems induced by low contrast boundaries. It is worth mentioning that the poor performance of FLUX in this experiment does not imply
FLUX is a not good algorithm for vascular segmentation. In fact, FLUX can well overcome low contrast and achieve a satisfiable segmentation result under a good initial condition. To prove this, we also present two experiments. In the first one, we change the value of $p$ in (26) from $99.9 \%$ to $99.5 \%$. This means the number of seeds is increased by 5 times. Different views of seeds of $p=99.5 \%$ are shown in the first row of Fig. 23. In this case, the segmentation result of FLUX becomes much better, as shown in the second row of Fig. 23. In the second one, we further relax the restriction on seed selection by uniformly sampling enough seeds in approximately all vessels according to the MIPs, in which case, the segmentation result of FLUX is further improved. 
Fig. 24 Two distinct views of the uniformly sampled seeds (the first row) and the segmentation result obtained by using FLUX (the second row)

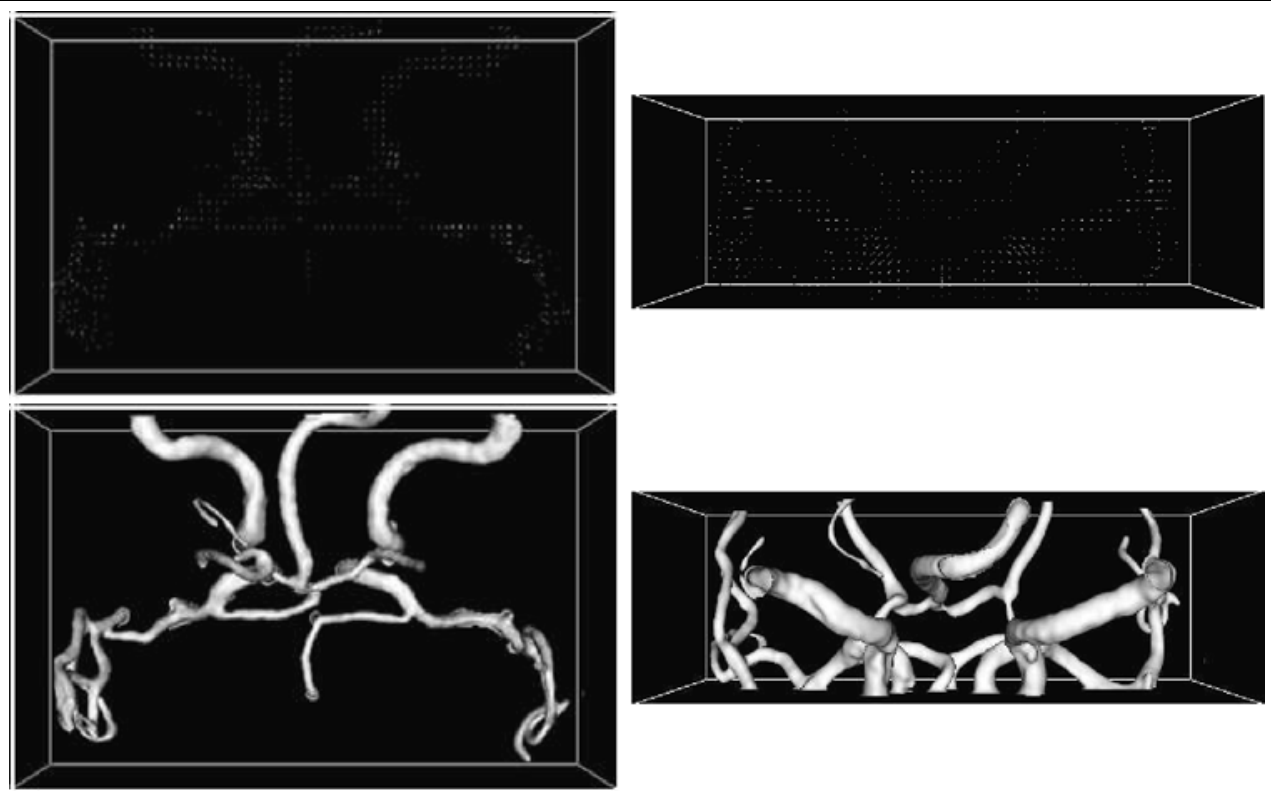

Two distinct views of the uniformly sampled seeds and the segmentation result of FLUX in this experiment are shown in Fig. 24.

In the second experiment, we test BWLSD-FLUX and compare it with FLUX on another $130 \times 286 \times 52$ brain PC-MRA image, which is named Image 2 in this paper. Because some low contrast boundaries are covered by other vessels in the top view, we also provide the rear view. Figures 25(a) and 25(b) respectively show the top and bottom views of the MIP of this PC-MRA image. These seeds are selected according to automatic intensity thresholding by setting $p=99.9 \%$ in (26). The top and bottom views of the seeds are displayed in Figs. 25(c) and 25(d), respectively. The top and bottom views of the segmentation result of FLUX are shown in Figs. 25(e) and 25(f), from which we can see that FLUX cannot propagate through several low contrast boundaries labeled from 1 to 7 in the MIPs when it starts evolution from these initial seeds as shown in Figs. 25(c) and 25(d). As a comparison, the segmentation result obtained by using BWLSD-FLUX is satisfactory, as shown in Fig. 25(i). Figures 25(e) and 25(f) are top and bottom views of one of the intermediate steps of BWLSDFLUX when the propagation contour has passed through the seven labeled low contrast regions. Similarly, we also provide two distinct views of MIP and the segmentation result of BWLSD-FLUX in Fig. 26.

Similar to the experiments on Image1, FLUX started from uniformly located seeds is also applied to Image2. Two distinct views of uniformly located seeds and the segmentation result of FLUX are shown in Fig. 27. Although worse than BWLSD-FLUX, FLUX in this case still achieves better results than FLUX started from the tough initial condition of $p=99.9 \%$. The segmentation result of FLUX can be further improved by increasing the number of seeds.
To further test our proposed method BWLSD-FLUX, a TOF-MRA image, which is named as Image3, is used in the third experiment. The top and bottom views of MIP of this image are displayed in Figs. 28(a) and 28(b), respectively. Started from seeds selected based on (26) with $p=99.9 \%$, as shown in Figs. 28(c) and 28(d), FLUX fails to pass through low contrast boundaries labeled by red dotted circles and consequently generates poor results, as shown in Figs. 28(e) and 28(f). In comparison with FLUX, two views of the segmentation result obtained by using BWLSDFLUX are exhibited in Figs. 28(g) and 28(h). The result shows that the low contrast boundaries labeled by red dotted circles are successfully passed through. Since the red dotted circles may cover a part of the vessels in Figs. 28(g) and 28(h), a more clear view of Figs. 28(g) and 28(h) with the removal of the red dotted circles are presented in the first row of Fig. 29. the corresponding views of MIP are shown in the second row of Fig. 29. The segmentation result of FLUX started from uniformly located seeds is displayed in Fig. 30. The uniformly located seeds are shown in this figure as well.

Image 4 is a PC-MRA image with size $120 \times 256 \times 58$ voxels. The MIPs of image4 are shown in the first row of Fig. 31. The corresponding views of the segmentation result of BWLSD-FLUX are shown in the second row of the figure. The seeds are selected automatically by setting $p=99.5 \%$, as shown in the first row of Fig. 32. Two different views of the segmentation result of FLUX with $\beta=0.05$ is shown in the second row of Fig. 32. Although more low contrast vessels can be segmented by FLUX with a smaller value of $\beta$, meanwhile the surface smoothness of the vessels reduces and the possibility of occurrence of leakages increases. As such, we should make a balance between accuracy and smoothness. Based on a large number 


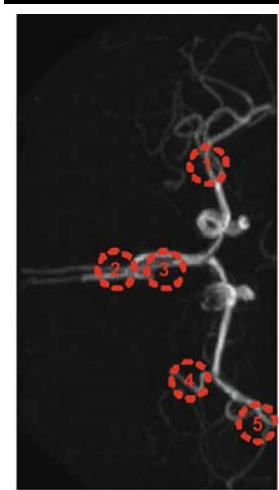

(a)

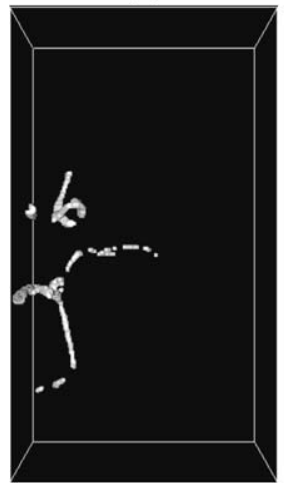

(d)

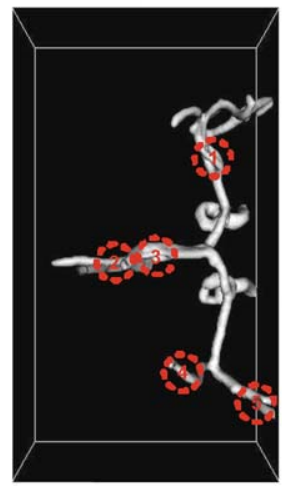

(g)

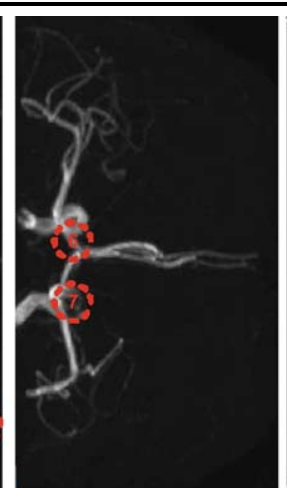

(b)

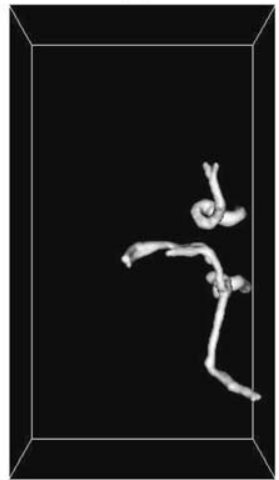

(e)

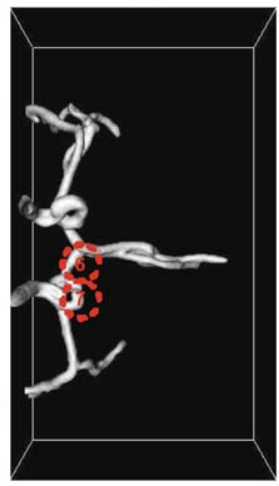

(h)

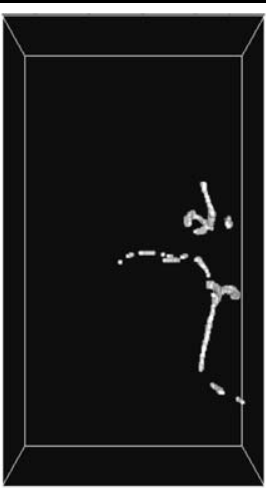

(c)

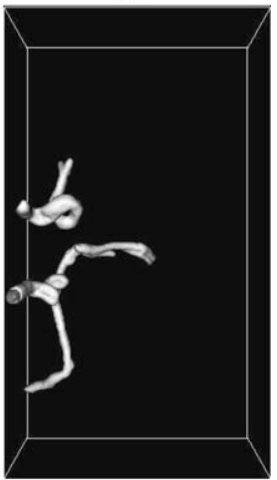

(f)

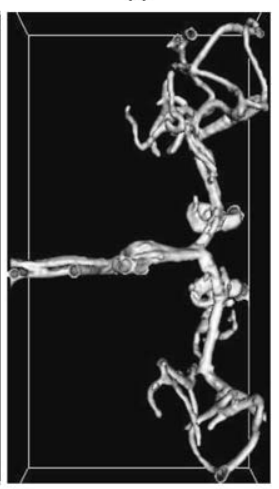

(i)
Fig. 25 An illustration of BWLSD-FLUX on segmentation of a $130 \times 286 \times 52$ brain PC-MRA image. (a) Top maximum intensity projection (MIP) view of the image. (b) Bottom MIP view of the image. (c) Top view of the initial seed image. (d) Bottom view of the initial seed image. (e) Top view of the segmentation result obtained by using FLUX. (f) Bottom view of the segmentation result obtained by using FLUX. (g) Top view of an evolution step of BWLSD-FLUX, where BWLSD-FLUX has propagated through the labeled low contrast boundaries. (h) Bottom view of this BWLSD-FLUX evolution step. (i) Segmentation result obtained by using BWLSD-FLUX

of experiments, we find that 0.05 is a proper value for $\beta$. Too large $\beta$ or too small $\beta$ will harm the segmentation result. The segmentation results of FLUX with $\beta=0$ and $\beta=0.01$ are shown in Figs. 33(a) and 33(b), respectively. From Fig. 33(b), it can be seen that the vessel surface obtained by FLUX with $\beta=0.01$ is much coarser and some low contrast vessels are not segmented. From Fig. 33(a), it can be observed that many leakages on the vessel surfaces obtained by FLUX with $\beta=0$ although more vessels are segmented.

A TOF-MRA image with size $115 \times 256 \times 60$ voxels, which is named Image 5 in this paper, is utilized in the fifth experiment. Two different MIP views of Image5 are shown in the first row of Fig. 34. Corresponding views of the segmentation results obtained by BWLSD-FLUX are shown in the second row of Fig. 34. Two views of the segmentation results obtained by FLUX are shown in the second row of Fig. 35. Both BWLSD-FLUX and FLUX start their evolutions from the same seeds, as selected automatically by setting $p=99.9 \%$, with $\beta=0.05$. Two views of the seeds are shown in the first row of Fig. 35. Obviously, BWLSDFLUX obtains a better segmentation result than FLUX in the low contrast vessels because of the ability of BWLSDFLUX against low contrast. The last experiment is carried out on a TOF-MRA image with size $110 \times 260 \times 64$ voxels, which is named Image6 in this paper. The MIP, initial seeds as well as the segmentation result of BWLSD-FLUX and FLUX are shown in Fig. 36. In this experiment, the seeds are selected automatically by setting $p=99.9 \%$, and the value of $\beta$ for BWLSD-FLUX and FLUX is 0.05 . The results of this experiment also illustrate BWLSD-FLUX is better than FLUX in segmenting low contrast vessels from the same initial seeds.

\section{Conclusions}

Our main contributions in this paper are two-fold. First, we design a new function (BWLSD) for measuring edge strength, which is capable of making the measured strengths of edges in regions with different contrasts and intensities more consistent. Second, we propose the criteria on determining the size of one local region by establishing the relationship between the local region size and the scale of wavelet transform. As far as we are concerned, though many algorithms using local image properties for image processing, including segmentation, enhancement, etc., have been proposed, most of them select the size of the local region empirically. Our attempt opens one door to solve this problem.

In the experiments on synthetic images, BWLSD illustrates its robustness against noise, inconsistent intensity and contrast by extracting high-quality edge maps from different types of images contaminated by both noise and intensity inhomogeneity. Based on BWLSD, we integrate it with FLUX to form a new segmentation algorithm, namely, BWLSDFLUX. Tested on three MRA image volumes, including PC-MRA and TOF-MRA, it is experimentally proved that BWLSD-FLUX is insensitive to intensity inhomogeneity, 
Fig. 26 Two distinct views of maximum intensity projection (the first row) and the segmentation result obtained by using BWLSD-FLUX (the second row)
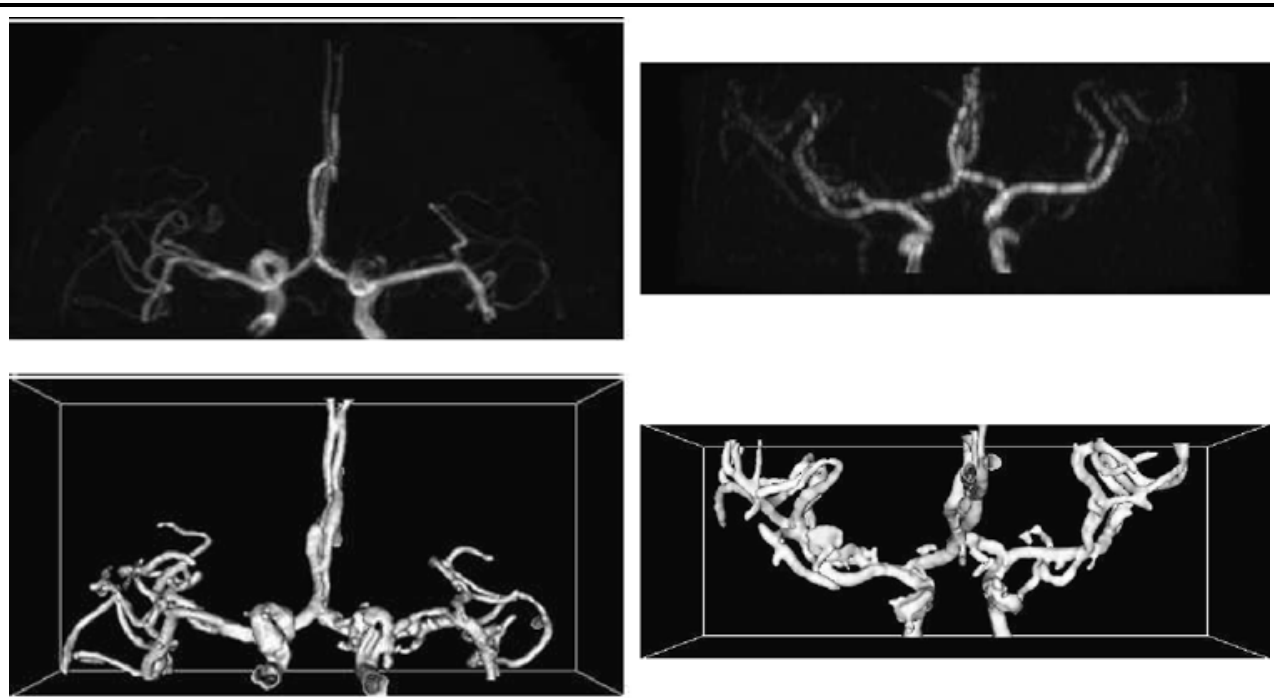

Fig. 27 Two distinct views of the uniformly sampled seeds (the first row) and the segmentation result obtained by using FLUX (the second row)
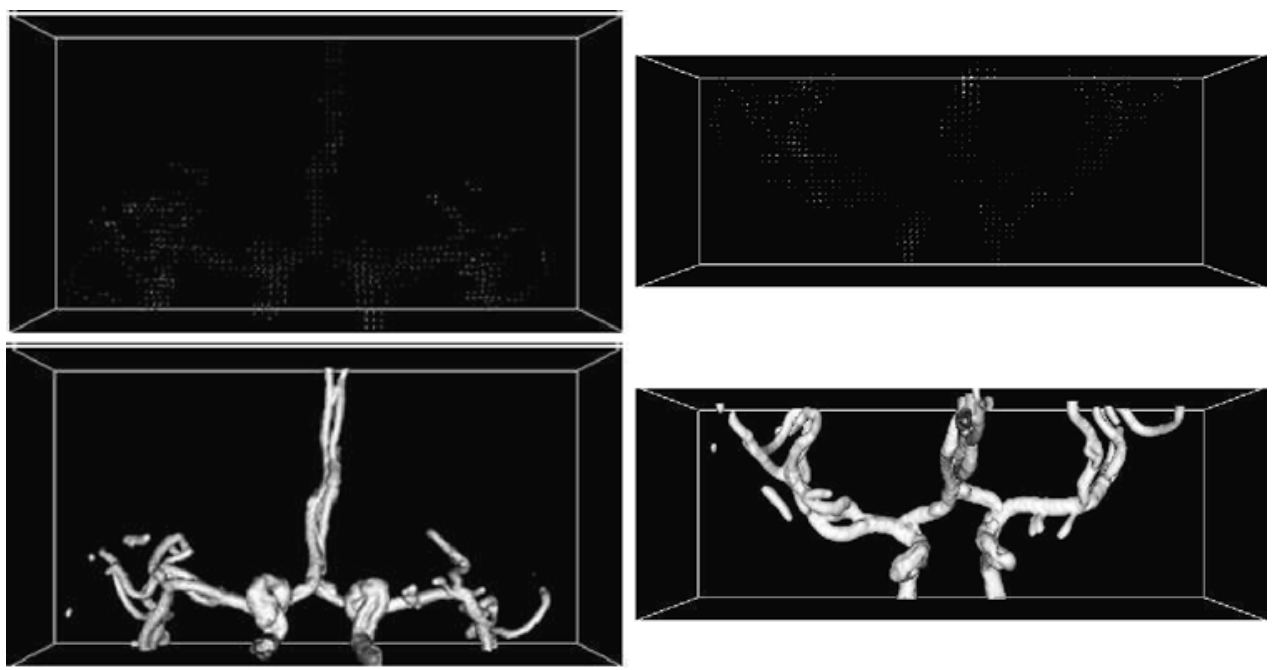

noise and other disturbances introduced by image acquisition system, and can supply satisfactory vascular segmentation results from MRA image volumes. In comparison with FLUX, BWLSD-FLUX reduces the requirement on initial condition and for this reason can be regarded as a remarkable improvement of FLUX.

Acknowledgements The authors would like to thank Dr. Paul Summers for providing the clinical data sets, and Mr. Max Law for providing the FLUX program for implementation and testing. The financial support from the K S Lo Foundation is gratefully acknowledged. The authors would also like to thank the anonymous reviewers for their constructive comments and suggestions.

\section{Appendix A: Proof of Lemma 1}

Lemma 1 The BWLSD values of two image edges with different contrasts and luminosities are equal.
Proof

$$
\begin{aligned}
G(B(\cdot)) & =\frac{\left|\nabla W_{s} f(B(\cdot))\right|}{\sigma\left(\left|\nabla W_{s} f(B)\right|\right)} \\
& =\frac{\sqrt{\sum_{i=1}^{3}\left|W_{s}^{i} f(B(\cdot))\right|^{2}}}{\sigma\left(\sqrt{\sum_{i=1}^{3}\left|W_{s}^{i} f(B)\right|^{2}}\right)} \\
& =\frac{\sqrt{\sum_{i=1}^{3}\left|s \frac{\partial}{\partial x_{i}}\left(f(B(\cdot)) * \theta_{s}\right)\right|^{2}}}{\sigma\left(\sqrt{\sum_{i=1}^{3}\left|s \frac{\partial}{\partial x_{i}}\left(f(B) * \theta_{s}\right)\right|^{2}}\right)} \\
& =\frac{\left.\sqrt{\sum_{i=1}^{3} \mid s \frac{\partial}{\partial x_{i}}((K f(A(\cdot))+C)} * \theta_{s}\right)\left.\right|^{2}}{\sigma\left(\sqrt{\sum_{i=1}^{3}\left|s \frac{\partial}{\partial x_{i}}\left((K f(A)+C) * \theta_{s}\right)\right|^{2}}\right)} \\
& =\frac{K \sqrt{\sum_{i=1}^{3}\left|s \frac{\partial}{\partial x_{i}}\left(f(A(\cdot)) * \theta_{s}\right)\right|^{2}}}{\sigma\left(K \sqrt{\sum_{i=1}^{3}\left|s \frac{\partial}{\partial x}\left(f(A) * \theta_{s}\right)\right|^{2}}\right)}
\end{aligned}
$$


Fig. 28 An illustration of BWLSD-FLUX on

segmentation of a $239 \times 209 \times 60$ brain TOF-MRA image. (a) Top maximum intensity projection (MIP) view of the image. (b) Bottom MIP view of the image. (c) Top view of the initial seed image. (d) Bottom view of the initial seed image. (e) Top view of the segmentation result obtained by using FLUX. (f) Bottom view of the segmentation result obtained by using FLUX. (g) Top view of the segmentation result obtained by using BWLSD-FLUX.

(h) Bottom view of the segmentation result obtained by using BWLSD-FLUX

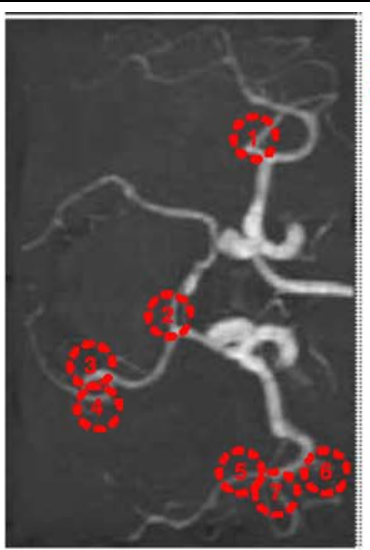

(a)

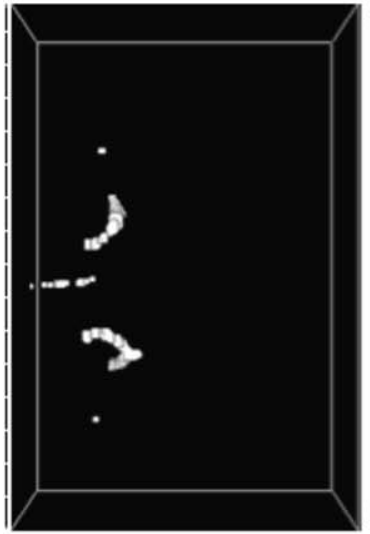

(d)

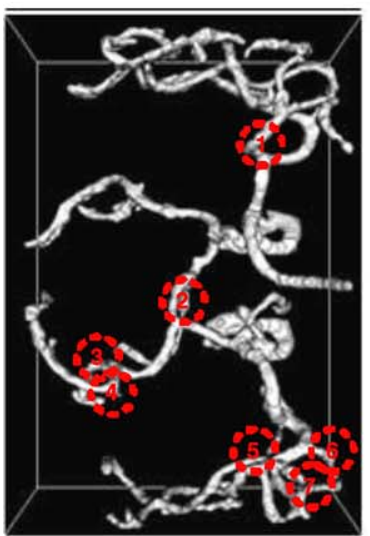

(g)

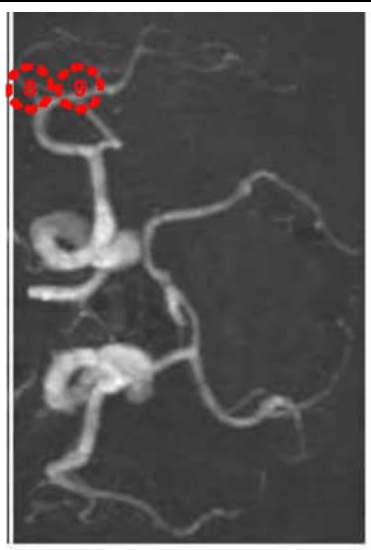

(b)

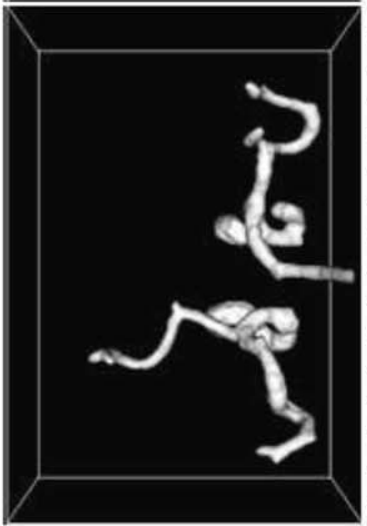

(e)

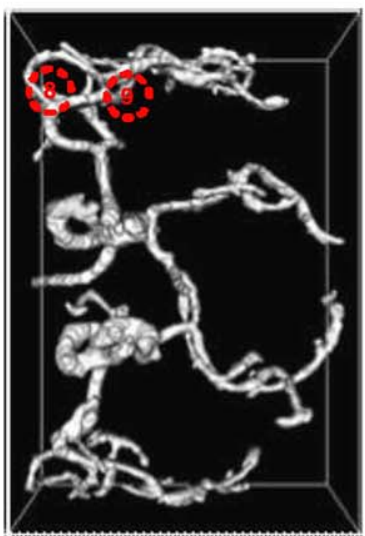

(h)

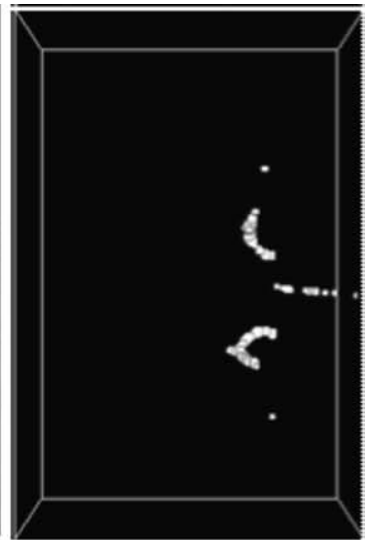

(c)

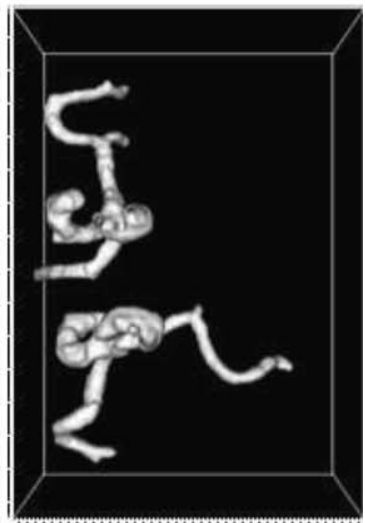

(f)

$$
\begin{aligned}
& =\frac{K\left(\sqrt{\sum_{i=1}^{3} \mid W_{s}^{i} f\left(\left.A(\cdot)\right|^{2}\right.}\right)}{K \sigma\left(\sqrt{\sum_{i=1}^{3}\left|W_{s}^{i} f(A)\right|^{2}}\right)} \\
& =\frac{\left|\nabla W_{s} f(A(\cdot))\right|}{\sigma\left(\left|\nabla W_{s} f(A)\right|\right)} \\
& =G(A(\cdot)) .
\end{aligned}
$$

The lemma is proved.

\section{Appendix B: Proof of Lemma 2}

Lemma 2 For a symmetric 3-D tube with radius $r$, the size of region with non-zero wavelet magnitude is $2 s-1$ on each side of the tube regardless of the direction and shape of the tube. $s$ is the scale of B-spline wavelet transform.

Proof A 3-D symmetric tube can be regarded as a set of points located within the circles around the center curve $C$ 
Fig. 29 The top and bottom views of the maximum intensity projection (the first row) and the corresponding views of the segmentation result obtained by using BWLSD-FLUX (the second row)
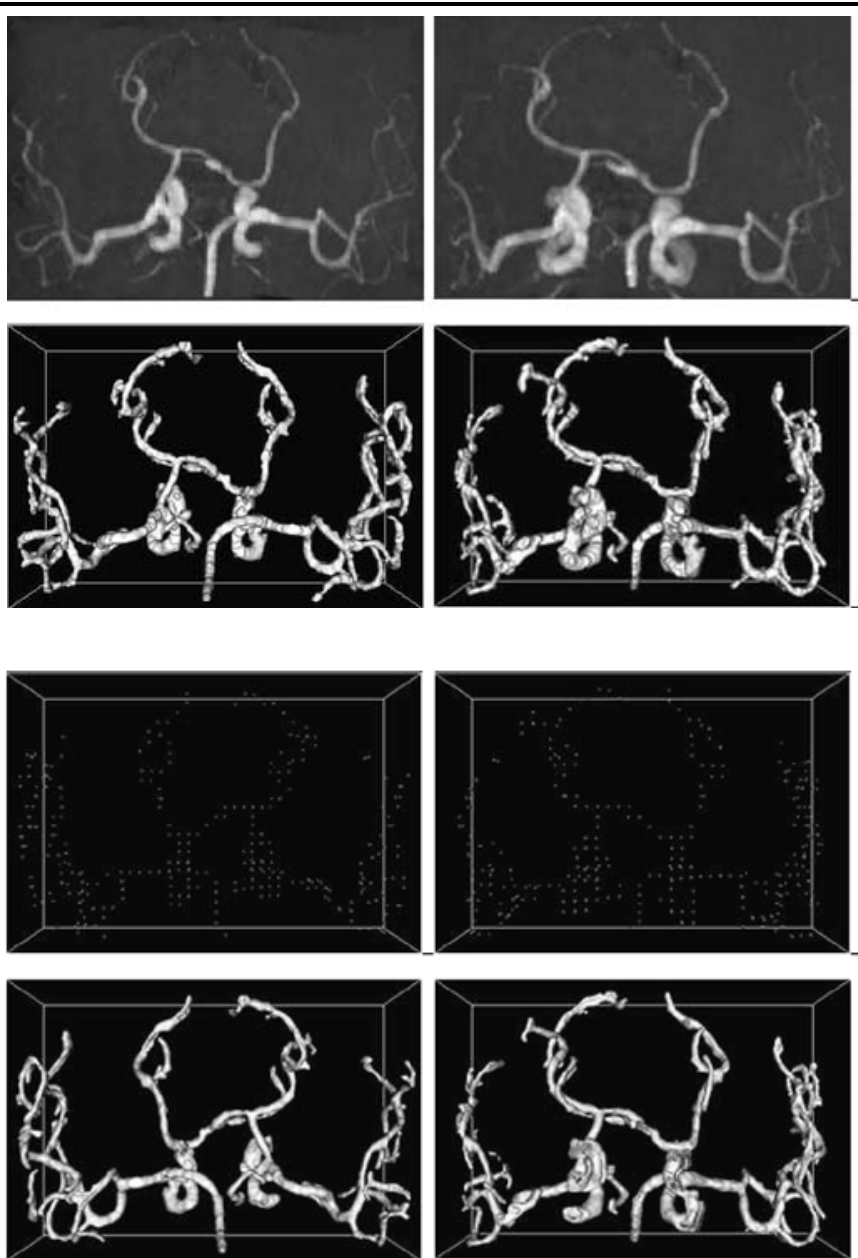

Fig. 31 Two distinct views of MIP (the first row) and the segmentation result obtained by using BWLSD-FLUX (the second row)
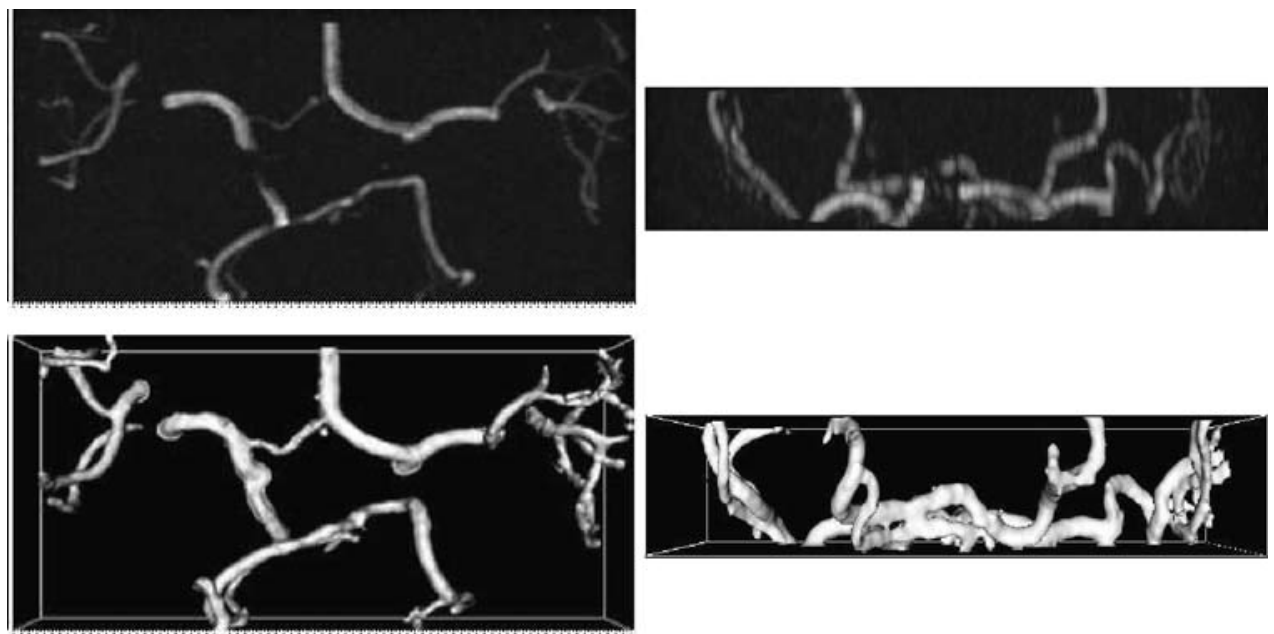

with radius $r$ ( $r$ can be a constant or variable), as seen in

Fig. 37. We only consider a local section of this curve, of which the start point, midpoint and end point are denoted as $c_{s}, c_{0}$ and $c_{e}$ respectively $\left(c_{s}<c_{0}<c_{e}\right)$. The coordinates of midpoint $c_{0}$ are $x_{0}, y_{0}$ and $z_{0}$. Accordingly, the coordinates of an arbitrary point $c\left(c \in\left[c_{s}, c_{e}\right]\right)$ of the center curve $C$ is represented by

$\left\{\begin{array}{l}x_{c}=x_{0}+\left(c-c_{0}\right) \cos \alpha_{1}, \\ y_{c}=y_{0}+\left(c-c_{0}\right) \cos \beta_{1}, \\ z_{c}=z_{0}+\left(c-c_{0}\right) \cos \gamma_{1},\end{array}\right.$ 
Fig. 32 Two distinct views of seeds of $p=99.5 \%$ (the first row) and the segmentation result obtained by using FLUX started from these seeds (the second row)
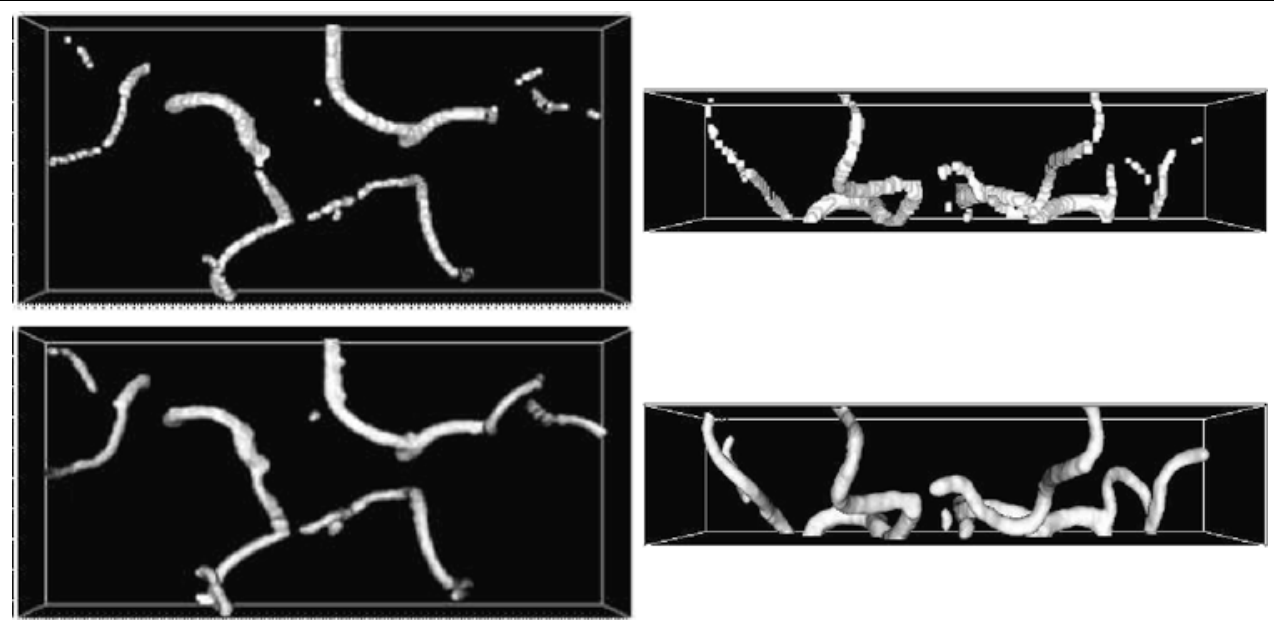

Fig. 33 Segmentation results obtained by using FLUX with a small $\beta$. (a) $\beta=0$. (b) $\beta=0.01$

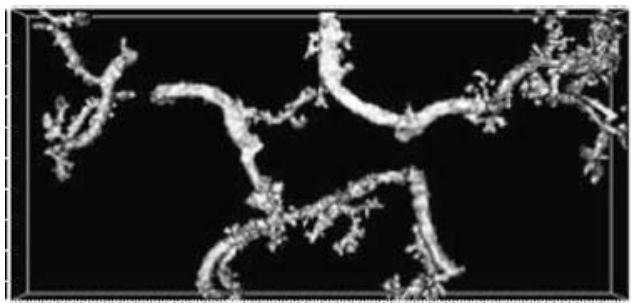

(a)
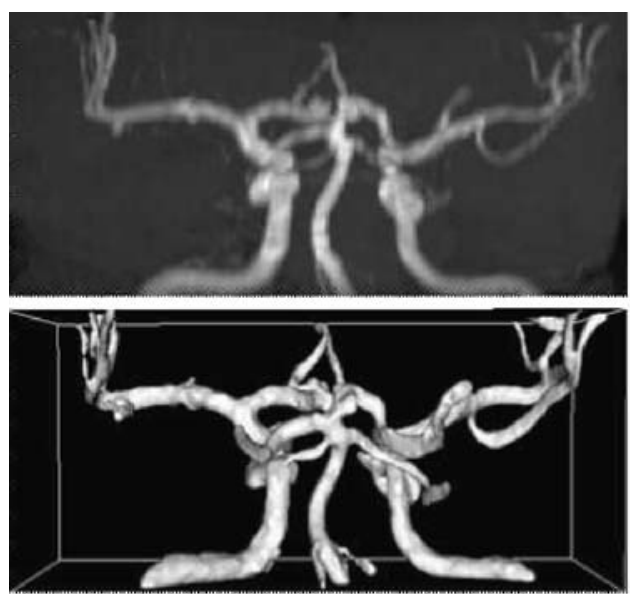

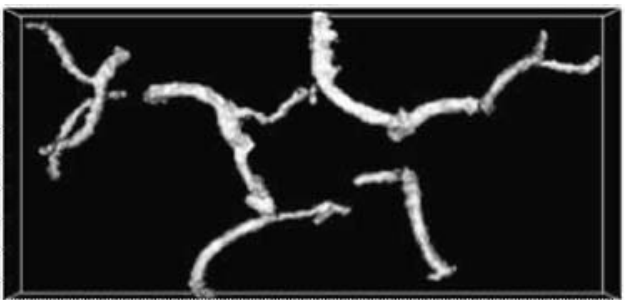

(b)
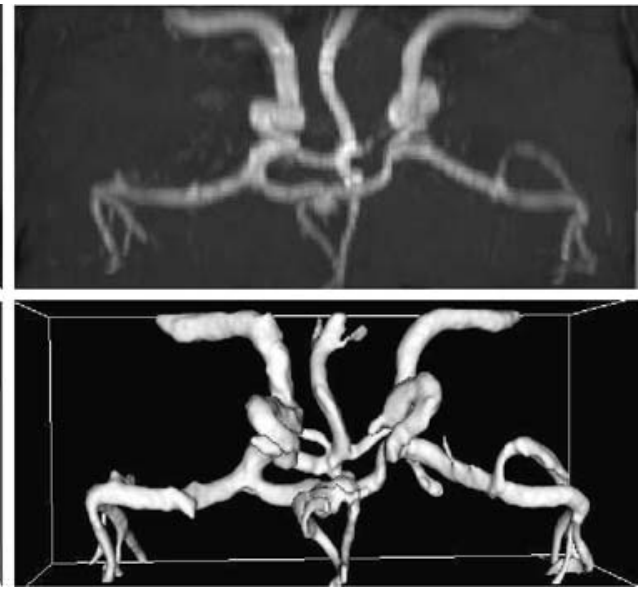

where $\cos \alpha_{1}, \cos \beta_{1}, \cos \gamma_{1}$ are the direction parameters of the line $\overrightarrow{c c_{0}}$. The parameter equation of the point $p$ located in the circle around the center point $c$ with radius $d$ can be written in form of

$\left\{\begin{array}{l}x_{p}=x_{d}(c)=x_{c}+d \cos \alpha_{2}, \\ y_{p}=y_{d}(c)=y_{c}+d \cos \beta_{2}, \\ z_{p}=z_{d}(c)=z_{c}+d \cos \gamma_{2},\end{array}\right.$

where $\cos \alpha_{2}, \cos \beta_{2}, \cos \gamma_{2}$ are the direction parameters of the line $\overrightarrow{p c}$ and $d$ is the distance of the line $\overrightarrow{p c}$. If the length of local section being taken into account is short enough, the line $\overrightarrow{c c_{0}}$ should be perpendicular to the radial line $\overrightarrow{p c}$. Thereafter, we obtain such a formula,

$\overrightarrow{c c_{0}} \cdot \overrightarrow{p c}=\cos \alpha_{1} \cos \alpha_{2}+\cos \beta_{1} \cos \beta_{2}+\cos \gamma_{1} \cos \gamma_{2}=0$

For simplicity, we use $\alpha_{1}, \beta_{1}, \gamma_{1}$ and $\alpha_{2}, \beta_{2}, \gamma_{2}$ to represent $\cos \alpha_{1}, \cos \beta_{1}, \cos \gamma_{1}$ and $\cos \alpha_{2}, \cos \beta_{2}, \cos \gamma_{2}$. In this way, (33) can be simplified as follows:

$\alpha_{1} \alpha_{2}+\beta_{1} \beta_{2}+\gamma_{1} \gamma_{2}=0$ 
Fig. 35 Two distinct views of seeds of $p=99.9 \%$ (the first row) and the segmentation result obtained by using FLUX started from these seeds (the second row)

Fig. 36 Another example by using a Tof-MRA image.

(a) MIP. (b) Seeds.

(c) Segmentation result obtained by BWLSD-FLUX.

(d) Segmentation result obtained by FLUX
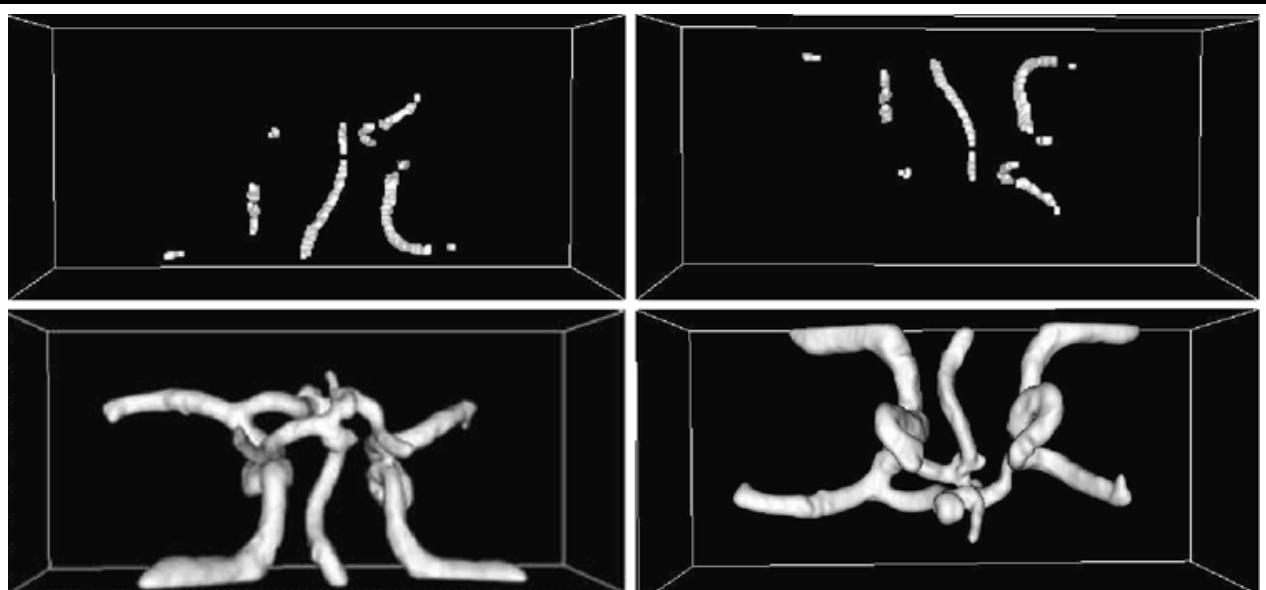

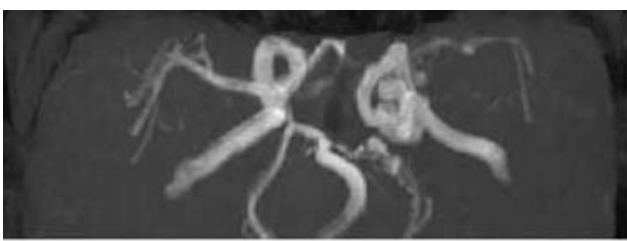

(a)

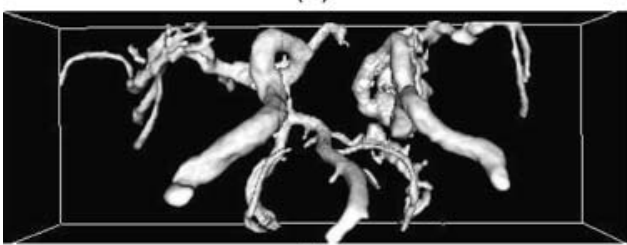

(c)

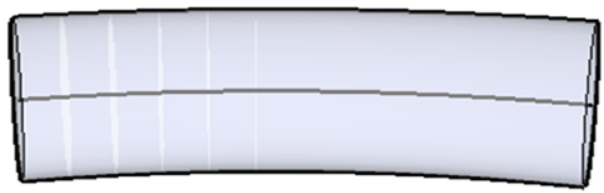

(a)

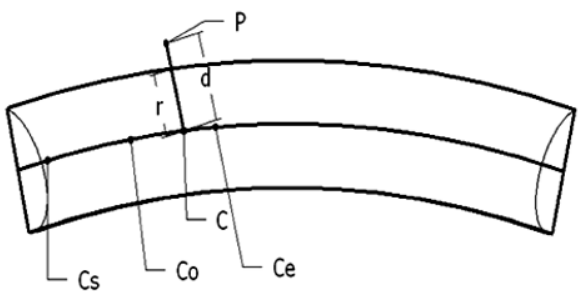

(c)

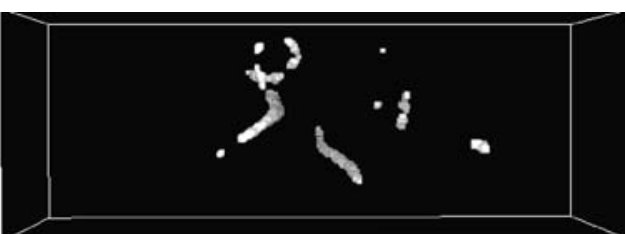

(b)

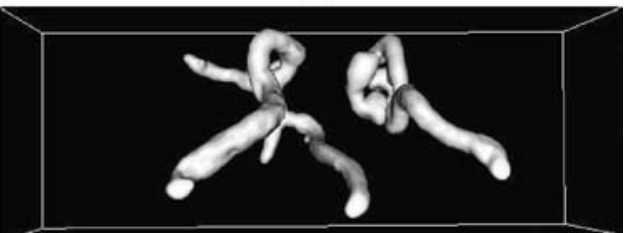

(d)
Fig. 37 Illustrative figures for the proof of Lemma 2. (a) A 3-D symmetric tube.

(b) Wavelet magnitude of the tube. The inner hollow tube (dotted pattern) is region with zero magnitude and the outer tube is the region with non-zero magnitude. (c) Cross section of the 3-D tube in (a). (d) Cross section of wavelet magnitude tube. The dotted region on both sides is the region with non-zero wavelet magnitude. The inner solid region is the region with zero wavelet magnitude

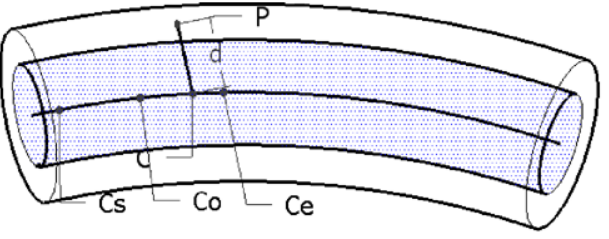

(b)

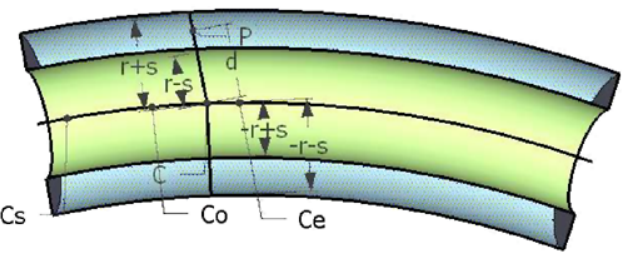

(d)
Note that, $\alpha_{1}, \beta_{1}, \gamma_{1}$ and $\alpha_{2}, \beta_{2}, \gamma_{2}$ also satisfy

$\left\{\begin{array}{l}\alpha_{1}^{2}+\beta_{1}^{2}+\gamma_{1}^{2}=1, \\ \alpha_{2}^{2}+\beta_{2}^{2}+\gamma_{2}^{2}=1 .\end{array}\right.$

We use the sign $f_{C, r}$ to denote a 3-D image which only contains one local tube segment whose center line is $\overrightarrow{c_{s} c_{e}}$ and radius is $r$. Without loss of generality, we define the intensity value of the points inside this tube as 1 and the points outside as 0 . Thus, for a point $p\left(x_{d}(c), y_{d}(c), z_{d}(c)\right)$ belonging to the image $f_{C, r}$, for $i=1,2,3$, the wavelet transform at scale $s$ can be expressed by 


$$
\begin{aligned}
& W_{s}^{i} f_{C, r}\left(\left(x_{d}(c), y_{d}(c), z_{d}(c)\right)\right. \\
& =\iiint_{R} f_{C, r}(x, y, z) \psi_{s}\left(x_{d}(c)-x, y_{d}(c)-y,\right. \\
& \left.z_{d}(c)-z\right) d x d y d z \\
& =\int_{c_{s}}^{c_{e}} d c \int_{-r}^{r} \psi_{s}\left(\alpha_{2}(d-\rho)-\alpha_{1}\left(c-c_{0}\right), \beta_{2}(d-\rho)\right. \\
& \left.\quad-\beta_{1}\left(c-c_{0}\right), \gamma_{2}(d-\rho)-\gamma_{1}\left(c-c_{0}\right)\right) d \rho .
\end{aligned}
$$

The smooth function $\theta$ defined by (1) can be extended to such a trivariate function

$\theta(x, y, z)=\phi\left(\sqrt{x^{2}+y^{2}+z^{2}}\right)$,

where

$\theta(r)= \begin{cases}8 r^{2}(r-1)+4 / 3, & r \in\left[0, \frac{1}{2}\right) ; \\ -8 / 3(r-1)^{3}, & r \in\left[\frac{1}{2}, 1\right), \\ 0, & r \in[1,+\infty) .\end{cases}$

By recalling the relationship between the wavelet function $\psi$ and the smooth function $\theta$ defined in (4), we have

$$
\left\{\begin{array}{l}
\psi^{1}(x, y, z)=\phi^{\prime}\left(\sqrt{x^{2}+y^{2}+z^{2}}\right) \frac{x}{\sqrt{x^{2}+y^{2}+z^{2}}}, \\
\psi^{2}(x, y, z)=\phi^{\prime}\left(\sqrt{x^{2}+y^{2}+z^{2}}\right) \frac{y}{\sqrt{x^{2}+y^{2}+z^{2}}}, \\
\psi^{3}(x, y, z)=\phi^{\prime}\left(\sqrt{x^{2}+y^{2}+z^{2}}\right) \frac{z}{\sqrt{x^{2}+y^{2}+z^{2}}} .
\end{array}\right.
$$

Consequently, the wavelet transform using the wavelet function $\psi^{1}$ is

$$
\begin{aligned}
& W_{s}^{1} f_{C, r}\left(\left(x_{d}(c), y_{d}(c), z_{d}(c)\right)\right. \\
& =\int_{c_{s}}^{c_{e}} d c \int_{-r}^{r} \psi_{s}\left(\alpha_{2}(d-\rho)-\alpha_{1}\left(c-c_{0}\right), \beta_{2}(d-\rho)\right. \\
& \left.\quad-\beta_{1}\left(c-c_{0}\right), \gamma_{2}(d-\rho)-\gamma_{1}\left(c-c_{0}\right)\right) d \rho \\
& =\int_{c_{s}}^{c_{e}} d c \int_{-r}^{r} \phi_{s}^{\prime}\left(\sqrt{(d-\rho)^{2}+\left(c-c_{0}\right)^{2}}\right. \\
& \left.\quad \times \frac{\alpha_{2}(d-\rho)-\alpha_{1}\left(c-c_{0}\right)}{\sqrt{(d-\rho)^{2}+\left(c-c_{0}\right)^{2}}}\right) d \rho \\
& =\int_{\left(c_{0}-c_{e}\right) / s}^{\left(c_{0}-c_{s}\right) / s} d c \int_{(d-r) / s}^{(d+r) / s} \phi^{\prime}\left(\sqrt{c^{2}+\rho^{2}}\right) \frac{\alpha_{2} \rho-\alpha_{1} c}{\sqrt{\rho^{2}+c^{2}}} d \rho .
\end{aligned}
$$

Similarly, the wavelet transforms using the wavelet functions $\psi^{2}, \psi^{3}$ are

$$
\begin{aligned}
& W_{s}^{2} f_{C, r}\left(\left(x_{d}(c), y_{d}(c), z_{d}(c)\right)\right. \\
& \quad=\int_{\left(c_{0}-c_{e}\right) / s}^{\left(c_{0}-c_{s}\right) / s} d c \int_{(d-r) / s}^{(d+r) / s} \phi^{\prime}\left(\sqrt{c^{2}+\rho^{2}}\right) \frac{\beta_{2} \rho-\beta_{1} c}{\sqrt{\rho^{2}+c^{2}}} d \rho,
\end{aligned}
$$

and

$$
\begin{aligned}
& W_{s}^{3} f_{C, r}\left(\left(x_{d}(c), y_{d}(c), z_{d}(c)\right)\right. \\
& \quad=\int_{\left(c_{0}-c_{e}\right) / s}^{\left(c_{0}-c_{s}\right) / s} d c \int_{(d-r) / s}^{(d+r) / s} \phi^{\prime}\left(\sqrt{c^{2}+\rho^{2}}\right) \frac{\gamma_{2} \rho-\gamma_{1} c}{\sqrt{\rho^{2}+c^{2}}} d \rho .
\end{aligned}
$$

For the sake of simplicity, we define two temporary variables

$T_{1}=\int_{\left(c_{0}-c_{e}\right) / s}^{\left(c_{0}-c_{s}\right) / s} d c \int_{(d-r) / s}^{(d+r) / s} \phi^{\prime}\left(\sqrt{c^{2}+\rho^{2}}\right) \frac{c}{\sqrt{\rho^{2}+c^{2}}} d \rho$,

and

$$
T_{2}=\int_{\left(c_{0}-c_{e}\right) / s}^{\left(c_{0}-c_{s}\right) / s} d c \int_{(d-r) / s}^{(d+r) / S} \phi^{\prime}\left(\sqrt{c^{2}+\rho^{2}}\right) \frac{\rho}{\sqrt{\rho^{2}+c^{2}}} d \rho .
$$

Then it is easy to represent $W_{s}^{i} f_{C r}\left(x_{d}(c), y_{d}(c), z_{d}(c)\right)$ in terms of $T_{1}$ and $T_{2}$,

$$
\left\{\begin{array}{l}
W_{s}^{1} f_{C, r}\left(\left(x_{d}(c), y_{d}(c), z_{d}(c)\right)=\alpha_{2} T_{2}-\alpha_{1} T_{1},\right. \\
W_{s}^{2} f_{C, r}\left(\left(x_{d}(c), y_{d}(c), z_{d}(c)\right)=\beta_{2} T_{2}-\beta_{1} T_{1},\right. \\
W_{s}^{3} f_{C, r}\left(\left(x_{d}(c), y_{d}(c), z_{d}(c)\right)=\gamma_{2} T_{2}-\gamma_{1} T_{1} .\right.
\end{array}\right.
$$

It follows that the square of the magnitude of the wavelet transform at point $p\left(x_{d}(c), y_{d}(c), z_{d}(c)\right)$ is

$$
\begin{aligned}
\mid \nabla & \left.W_{s} f_{C r}\left(x_{d}(c), y_{d}(c), z_{d}(c)\right)\right|^{2} \\
= & \sum_{i=1}^{3}\left(W_{s}^{i} f_{C, r}\left(x_{d}(c), y_{d}(c), z_{d}(c)\right)\right)^{2} \\
= & \left(\alpha_{2} T_{2}-\alpha_{1} T_{1}\right)^{2}+\left(\beta_{2} T_{2}-\beta_{1} T_{1}\right)^{2}+\left(\gamma_{2} T_{2}-\gamma_{1} T_{1}\right)^{2} \\
= & \left(\alpha_{2}^{2}+\beta_{2}^{2}+\gamma_{2}^{2}\right) T_{2}^{2}-\left(2 \alpha_{1} \alpha_{2}+2 \beta_{1} \beta_{2}+2 \gamma_{1} \gamma_{2}\right) T_{1} T_{2} \\
& \quad+\left(\alpha_{1}^{2}+\beta_{1}^{2}+\gamma_{1}^{2}\right) T_{1}^{2} \\
= & T_{1}^{2}+T_{2}^{2} .
\end{aligned}
$$

As we can see from (44), the direction parameters $\alpha_{1}, \beta_{1}$, $\gamma_{1}$ and $\alpha_{2}, \beta_{2}, \gamma_{2}$ disappear in the final result. This means the magnitude of the wavelet transform is direction-free. The result in (44) is also shape-free since it is based on the analysis on a local shape section.

Next, we continue to mathematically analyze the magnitude of the wavelet transform based on (44). We can rewrite the definition function of $T_{1}$ in (41) as

$$
T_{1}=\int_{(d-r) / s}^{(d+r) / s} d \rho \int_{\left(c_{0}-c_{e}\right) / s}^{\left(c_{0}-c_{s}\right) / s} \phi^{\prime}\left(\sqrt{\rho^{2}+c^{2}}\right) \frac{c}{\sqrt{\rho^{2}+c^{2}}} d c .
$$

Since the second integral function $\phi^{\prime}\left(\sqrt{\rho^{2}+c^{2}}\right) \frac{c}{\sqrt{\rho^{2}+c^{2}}}$ is an odd function in terms of variable $c$ and the integral field 
$\left[\left(c_{0}-c_{e}\right) / s,\left(c_{0}-c_{s}\right) / s\right]$ is symmetric on the original point, it is apparent that the result of (45) is 0 . Hence, the magnitude of wavelet transform becomes:

$$
\begin{aligned}
\mid \nabla & W_{s} f_{C, r}\left(x_{d}(c), y_{d}(c), z_{d}(c)\right) \mid \\
& =\left|T_{2}\right| \\
& =\int_{\left(c_{0}-c_{e}\right) / s}^{\left(c_{0}-c_{s}\right) / s} d c \int_{(d-r) / s}^{(d+r) / s} \phi^{\prime}\left(\sqrt{c^{2}+\rho^{2}}\right) \frac{\rho}{\sqrt{\rho^{2}+c^{2}}} d \rho .
\end{aligned}
$$

If we define $g$ is the distance between the midpoint $c_{0}$ to the start point $c_{s}$ and the end point $c_{e}$, that is, $g=c_{0}-c_{s}=$ $c_{e}-c_{0},(46)$ can be rewritten as

$$
\begin{aligned}
\mid \nabla & W_{s} f_{C, r}\left(x_{d}(c), y_{d}(c), z_{d}(c)\right) \mid \\
= & \left|\int_{-g / s}^{g / s} d c \int_{(d-r) / S}^{(d+r) / s} \phi^{\prime}\left(\sqrt{\rho^{2}+c^{2}}\right) \frac{\rho}{\sqrt{\rho^{2}+c^{2}}} d \rho\right| \\
= & \left|\int_{-g / S}^{g / S} d c \int_{\sqrt{c^{2}+((d-r) / s)^{2}}}^{\sqrt{c^{2}+((d+r) / s)^{2}}} \phi^{\prime}(\rho) d \rho\right| \\
= & 2 \mid \int_{0}^{g / s}\left(\phi\left(\sqrt{c^{2}+((d+r) / s)^{2}}\right)\right. \\
& \left.-\phi\left(\sqrt{c^{2}+((d-r) / s)^{2}}\right)\right) d c \mid .
\end{aligned}
$$

From the definition of $\phi(r)$ given in (38), we easily see that $\phi(r)=0$ when $r \geq 1$. Thus, to guarantee the value of the magnitude of wavelet transform $\mid \nabla W_{s} f_{C, r}\left(x_{d}(c), y_{d}(c)\right.$, $\left.z_{d}(c)\right) \mid$ is non-zero, $d$, the distance between the point $p\left(x_{d}(c), y_{d}(c), z_{d}(c)\right)$ to the corresponding center point $c$, should satisfy

$r-s<d<r+s$,

or

$-r-s<d<-r+s$.

The (+) and (-) signs of $r$ refer to different sides of the center line. For a discrete image, the discrete value of $d$ should lie within such intervals $[r-s+1, r+s-1]$ and $[-r-s+1,-r+s-1]$. Therefore, for a symmetric 3-D tube with radius $r$, the width of region with non-zero wavelet magnitude is $2 s-1$ on all sides of the tube regardless of the direction and shape of the tube.

\section{Appendix C: Proof of Lemma 3}

Lemma 3 For an asymmetric 3-D tube with radii $r$, the size of region with non-zero wavelet magnitude is $2 s-1$ on each side of the tube regardless of the direction and shape of the tube. $s$ is the scale of $B$-spline wavelet transform.

Proof The differences between asymmetric and symmetric 3-D tubes are that all sides of the symmetric 3-D tube have the same radius while different sides of the asymmetric 3-D tube have dissimilar radii which are denoted as $\left\{r_{1}, r_{2}, \ldots, r_{n}\right\}$. If the radius along the direction of $\overrightarrow{p c}$ is $r_{k}, k \in\{1, \ldots, n\}$, then (36) is changed as

$$
\begin{aligned}
& W_{s}^{i} f_{C, r}\left(\left(x_{d}(c), y_{d}(c), z_{d}(c)\right)\right. \\
& \quad=\iiint_{R} f_{C, r}(x, y, z) \psi_{s}\left(x_{d}(c)-x, y_{d}(c)-y\right. \\
& \left.z_{d}(c)-z\right) d x d y d z \\
& \quad=\int_{c_{s}}^{c_{e}} d c \int_{0}^{r_{k}} \psi_{s}\left(\alpha_{2}(d-\rho) \beta_{2}(d-\rho)-\beta_{1}\left(c-c_{0}\right)\right. \\
& \left.\quad-\alpha_{1}\left(c-c_{0}\right), \gamma_{2}(d-\rho)-\gamma_{1}\left(c-c_{0}\right)\right) d \rho
\end{aligned}
$$

Equation (47) is changed accordingly as

$$
\begin{aligned}
& \left|\nabla W_{s} f_{C, r}\left(x_{d}(c), y_{d}(c), z_{d}(c)\right)\right| \\
& \quad=2\left|\int_{0}^{g / s} \phi\left(\sqrt{c^{2}+\left(\left(d-r_{k}\right) / s\right)^{2}}\right) d c\right| .
\end{aligned}
$$

Other parts of the prove procedure are similar as that of Lemma 2.

At last, to ensure the wavelet magnitude $\mid \nabla W_{s} f_{C, r}\left(x_{d}(c)\right.$, $\left.y_{d}(c), z_{d}(c)\right) \mid$ is not zero, $d$ should satisfy

$r_{k}-s<d<r_{k}+s$.

Therefore, as a conclusion, for an asymmetric 3-D tube, the width of region with non-zero wavelet magnitude is also $2 s-1$ on all sides of the tube regardless of the direction and shape of the tube.

\section{Appendix D: Comparison with WLV}

Recently, Law and Chung proposed a weighted local variance-based (WLV) edge detection method to address the problem of changes of intensity contrast of edges (Law and Chung 2007). Although WLV is capable of providing high detection responses on low contrast edges, it suffers from three drawbacks.

First, in WLV, the 2-D or 3-D directional DOG filter $f_{\hat{n}}(\mathbf{x})$ is firstly split into two symmetrical halves, $f_{1, \hat{n}}(\mathbf{x})$ and $f_{2, \hat{n}}(\mathbf{x})$. Here, $\hat{n}$ refers to the direction of the directional DOG filter. Then the two halves $f_{i, \hat{n}}(\mathbf{x}), i=\{1,2\}$ are normalized into $g_{i, \hat{n}}(\mathbf{x})$ to let the sum of their coefficients be one. Afterward, WLVs are calculated by convolving the image $I(\mathbf{x})$ with the filters $g_{i, \hat{n}}(\mathbf{x}), W L V_{i, \hat{n}}(\mathbf{x})=$ 
$\int g_{i, \hat{n}}\left(I(\mathbf{x}+\mathbf{y})-\mu_{i, \hat{n}}(\mathbf{x})\right)^{2} d \mathbf{y}$. The measurement function for edge strength, $R_{\hat{n}}(\mathbf{x})$, is then defined as, $R_{\hat{n}}(\mathbf{x})=$ $\frac{\mu_{1, \hat{n}}(\mathbf{x})-\mu_{2, \hat{n}}(\mathbf{x})}{\sqrt{\min \left(W L V_{1, \hat{n}}, W L V_{2, \hat{n}}\right)+\epsilon}}$. Here, $\mu_{i, \hat{n}}$ are the weighted intensity averages of their corresponding filters $g_{i, \hat{n}}$, and $\epsilon$ is a small positive number to avoid division by zero. Therefore, to correctly measure the edge strength of one point $\mathbf{x}$, an edge across $\mathbf{x}$ is required to split the DOG filter $f_{\hat{n}}(\mathbf{x})$ into two symmetrical halves. Unfortunately, for points at one edge with a high curvature, such as corner points, there does not exist such an edge across them to symmetrically split the DOG filter. As such, the edge strength of corner points cannot be correctly measured in WLV. In BWLSD, the BWLSD function (see (12)) to measure the edge strength of one point is based on 1-D convolution and does not exist the problem of splitting one 2-D or 3-D filter. Therefore, the BWLSD function has a better performance than WLV on measuring the edge strength of corner points.

Second, WLV is based on one directional DOG filter $f_{\hat{n}}(\mathbf{x})$ and the WLV value of one point varies along the direction of the DOG filter. As such, a drawback in WLV is to find the direction of the DOG filter, along which the WLV confidence value can represent the edge strength of one point. This direction is also the edge normal direction. To estimate this direction, an image should be convolved with the DOG filter many times along a set of discretized directions. Typically, convolutions along 24 discretized directions should be made for one 2-D image and convolutions along 282 discretized directions should be made for one 3-D image. After obtaining the WLV confidence values in different discretized directions, an eigen-decomposition on one matrix associated with these WLV confidence values is performed to find the eigenvalues and eigenvectors of this matrix. The direction of the eigenvector associated with the largest eigenvalue is used as the edge normal direction. While in BWLSD, considering multi-dimensional B-spline smooth function and wavelet function are separable, the 2-D or 3-D wavelet transforms are turned into 1-D convolutions using 1-D B-spline smooth function and wavelet function. After obtaining the results of the wavelet transform along $x, y$ directions by convoluting the image with the wavelet filters four times (for one 2-D image) or $x, y, z$ directions by convoluting the image with the wavelet filters nine times (for one 3-D image), the edge strength measured by BWLSD function as well as the edge normal direction can be easily computed. Hence, BWLSD is implemented much faster than WLV.

Third, in WLV, the size of a local region to provide the estimation of local variance is selected empirically. This selection is lack of the theoretical support. While in BWLSD, we establish the theoretical relationship between the scale of the wavelet function and the size of the local region with nonzero wavelet magnitudes. Based on this relationship, we find that, if the scale of the wavelet function is $s$, then the size of one local region to provide the estimation of local standard derivation should be $2 s-1$. This relationship between the wavelet scale and the size of a local region is well testified by the our experiments on synthetic images and clinical images. This relationship is also accord with our experience. That is, if the image quality is poor, a large wavelet scale is used. Accordingly, a large image region is needed to provide a reliable estimation of the standard deviation, and vice verse.

In summary, BWLSD is obviously different from WLV and overcomes several drawbacks of WLV.

\section{References}

AIHW (2004). Heart, stroke and vascular diseases, Australian facts 2004. Australian Institute of Health and Welfare, Australian Government. http://www.aihw.gov.au/publications/cvd/hsvd04/ hsvd04.pdf.

Ambrosio, L., \& Soner, H. (1996). Level set approach to mean curvature flow in arbitrary codimension. Journal of Differential Geometry, 41(4), 693-737.

Armande, N., Montesinos, P., \& Monga, O. (1999). Thin nets extraction using multi-scale approach. Computer Vision and Image Understanding, 73(2), 248-257.

Aylward, S., \& Bullitt, E. (2002). Initialization, noise, singularities, and scale in height ridge traversal for tubular object centerline extraction. IEEE Transactions on Medical Imaging, 21(2), 61-75.

Bombardier, V., Jaluent, M., Bubel, A., \& Bermont, J. 1997. Cooperation of two fuzzy segmentation operators for digital subtracted angiograms analysis. In Proceedings of IEEE conference on fuzzy systems (pp. 1057-1062).

Bullitt, E., Aylward, S., Smith, K., Mukherji, S., Jiroutek, M., \& Muller, K. (2001). Symbolic description of intracerebral vessels segmented from MRA and evaluation by comparison with X-ray angiograms. IEEE Transactions on Medical Imaging, 5, 157-169.

Canny, J. (1986). A computational approach to edge detection. IEEE Transactions on Pattern Analysis and Machine Intelligence, 8(6), 679-698.

Caselles, V., Kimmel, R., \& Sapiro, G. (1997). Geodesic active contours. International Journal of Computer Vision, 22(1), 61-79.

CDC (2008). Chronic disease overview. Centers for Disease Control and Prevention. http://www.cdc.gov/nccdphp/overview.htm.

Chen, J., Sato, Y., \& Tamura, S. (1998). Orientation space filtering for multiple orientation line segmentation. In Proceedings of IEEE conference on computer vision and pattern recognition (pp. 311317).

Chwialkowski, M., Ibrahim, Y., \& Peshock, R. (1996). A method for fully automated quantitative analysis of arterial flow using flow-sensitized MR images. Computerized Medical Imaging and Graphics, 20(5), 365-378.

Cohen, I., \& Cohen, L. (1993). Finite-element methods for active contour models and balloons for 2-d and 3-d images. IEEE Transactions on Pattern Analysis and Machine Intelligence, 15, 11311147.

Duncan, J., \& Ayache, N. (2000). Medical image analysis: progress over two decades and the challenges ahead. IEEE Transactions on Pattern Analysis and Machine Intelligence, 22(1), 85-106.

Eiho, S., \& Qian, Y. (1997). Detection of coronary artery tree using morphological operator. IEEE Computers in Cardiology, 24, 525528.

Figueiredo, M., \& Leitao, J. (1995). A nonsmoothing approach to the estimation of vessel contours in angiograms. IEEE Transactions on Medical Imaging, 14, 162-172. 
Foracchia, M., Grisan, E., \& Ruggeri, A. (2003). Luminosity and contrast normalization in retinal images. Medical Image Analysis, 9, 179-190.

Frangi, A., Niessen, W., \& Viergever, M. 1998. Multiscale vessel enhancement filtering. In Proceedings of international conference on medical image computing and computer-assisted intervention (pp. 130-137).

Gooya, A., Liao, H., Matsumiya, K. K., Masamune, Y. M., \& Dohi, T. (2008). A variational method for geometric regularization of vascular segmentation in medical images. IEEE Transactions on Medical Imaging, 17(8), 1295-1312.

Guo, D., \& Richardson, P. (1998). Automatic vessel extraction from angiogram images. IEEE Computers in Cardiology, 25, 441-444.

He, J., Gu, D., Wu, X., Reyonlds, K., Duan, X., Yao, C., Wang, J., Chen, C., Chen, J., Wildman, R., Klag, M., \& Whelton, P. (2005). Major causes of death among men and women in china. The New England Journal of Medicine, 353(11), 1124-1134.

Higgins, W., Spyra, W., Karwoski, R., \& Ritman, E. (1996). System for analyzing high resolution three-dimensional coronary angiograms. IEEE Transactions on Medical Imaging, 15(3), 377385.

Holtzman-Gazit, M., Kimmel, R., Peled, N., \& Goldsher, D. (2006). Segmentation of thin structures in volumetric medical images. IEEE Transactions on Image Processing, 15(2), 354-363.

Hoover, A., Kouznetsova, V., \& Goldbaum, M. (2000). Locating blood vessels in retinal images by piecewise threshold probing of a matched filter response. IEEE Transactions on Medical Imaging, 19(3), 203-210.

Huang, Q., \& Stockman, F. (1993). Generalized tube model: recognizing 3-d elongated objects from 2-d intensity images. In Proceedings of the IEEE conference on computer vision and pattern recognition (pp. 104-109).

Hunter, I., Soraghan, J., \& McDonagh, T. (1995). Fully automatic left ventricular boundary extraction in echocardiographic images. IEEE Computers in Cardiology, 741-744.

Ibanez, L., Schroeder, W., Ng, L., \& Cates, J. (2005). The ITK software guide: the insight segmentation and registration toolkit (2nd ed.). Kitware.

Kass, M., Witkin, A., \& Terzopoulos, D. (1988). Snakes: active contour models. International Journal of Computer Vision, 1(4), 15731405.

Kirbas, C., \& Quek, F. (2004). A level-set approach to 3-d reconstruction from range data. ACM Computing Surveys, 36(2), 81-121.

Krissian, K., Malandain, G., \& Ayache, N. (1996). Directional anisotropic diffusion applied to segmentation of vessels in 3-d images. In Proceedings of the first conference on scale-space theory in computer vision (pp. 345-348).

Law, M., \& Chung, A. (2007). Weighted local variance-based edge detection and its application to vascular segmentation in magnetic resonance angiography. IEEE Transactions on Medical Imaging on Mathematical Modeling in Biomedical Image Analysis (Special issue), 26(9), 1224-1241.

Leroy, B., Herlin, I., \& Cohen, L. (1996). Multiresolution algorithms for active contour models. In Proceedings of international conference on analysis and optimization of system (pp. 58-65).

Lorigo, L., Faugeras, O., Grimson, W., Keriven, R., Kikinis, R., Nabavi, A., \& Westin, C. (2001). Curves: curve evolution for vessel segmentation. Medical Image Analysis, 5(3), 195-206.

Malladi, R., Sethian, J., \& Vemuri, B. (1995). Shape modeling with front propagation: a level set approach. IEEE Transactions on Pattern Analysis and Machine Intelligence, 17(2), 158-175.

Mallat, S. (1998). Wavelet tour of signal processing. San Diego: Academic Press.

Mallat, S., \& Hwang, W. (1992). Singularity detection and processing with wavelets. IEEE Transactions on Information Theory, 38 , 617-643.
Mallat, S., \& Zhong, S. (1992). Characterization of signals from multiscale edges. IEEE Transactions on Pattern Analysis and Machine Intelligence, 14, 710-732.

McInerney, T., \& Terzopoulos, D. (2000). T-snakes: topology adaptive snakes. Medical Image Analysis, 4(2), 73-91.

Mendonca, A., \& Campilho, A. (2006). Segmentation of retinal blood vessel by combining the detection of centerlines and morphological reconstruction. IEEE Transactions on Medical Imaging, 25(9), $1200-1213$.

O'Brien, J., \& Ezquerra, N. (1994). Automated segmentation of coronary vessels in angiographic image sequences utilizing temporal, spatial structural constraints. In Proceedings of the conference on visualization in biomedical computing (Vol. 25, pp. 25-37).

Osher, S., \& Sethian, J. (1988). Fronts propagating with curvature dependent speed: algorithm based on Hamilton-Jacobi formulations. Journal of Computational Physics, 79(1), 12-49.

Parvin, B., Penf, C., Johnston, W., \& Maestre, F. (1994). Tracking of tubular objects for scientific application. In Proceedings of IEEE conference on computer vision and pattern recognition (pp. 295301).

Polli, R., \& Valli, G. (1997). An algorithm for real-time vessel enhancement and detection. Computer Methods and Programs in Biomedicine, 52, 1-22.

Prinet, V., Monga, O., \& Rocchisani, J. (1995). Multi-dimensional vessel extraction using crest lines. In Proceedings of IEEE 17th conference on engineering in medicine and biology (pp. 393-394).

Quek, F., Kirbas, C., \& Charbel, F. (1999). Aim: attentionally-based interaction model for the interpretation of vascular angiograph. IEEE Transactions on Information Technology in Biomedicine, 3(2), 139-150.

Rekovei, R., \& Sun, Y. (1995). Back-propagation network and its configuration for blood vessel detection in angiograms. IEEE Transactions on Neural Networks, 6(1), 64-72.

Rinkel, G., Djibuti, M., Algra, A., \& Gijn, J. (1998). Prevalence and risk of rupture of intracranial aneurysms: a systematic review. Stroke, 29, 251-256.

Sato, Y., Nakajima, S., Atsumi, N., Yoshida, H., Koller, S., \& Gerig, T. (1998). Three-dimensional multiscale line filter for segmentation and visualization of curvilinear structures in medical images. Medical Image Analysis, 2(2), 143-168.

Schmitt, H., Grass, M., Rasche, V., Schermm, O., Haehnel, S., \& Sartor, K. (2002). An X-ray-based method for the determination of the contrast agent propagation in 3-d vessel structures. IEEE Transactions on Medical Imaging, 21(3), 251-262.

Sethian, J. (1996). A fast marching level set method for monotonically advancing fronts. Proceedings of the National Academy of Sciences of the United States of America, 93, 1591-1594.

Smets, C., Verbeeck, G., Suetens, P., \& Osterlinck, A. (1988). A knowledge-based system for the delineation of blood vessels on subtraction angiograms. Pattern Recognition Letters, 8, 113121.

Sorantin, E., Halmai, C., Erdohelyi, B., Palagyi, K., Olle, L., Geiger, K., Friedrich, F. L. G., \& Kiesler, K. (2002). Spiral-ct-based assessment of tracheal stenoses using 3-d skeletonization. IEEE Transactions on Medical Imaging, 21(3), 263-273.

Staal, J., Abramoff, M., Niemeijer, M., Viergever, M., \& Ginneken, B. (2004). Ridge-based vessel segmentation in color image of the retina. IEEE Transactions on Medical Imaging, 23(23), 501-509.

Tang, Y., Yang, L., \& Liu, J. (2000). Characterization of Dirac edge with new wavelet transform. IEEE Transactions on System, Man and Cybernetics Part B, 30(1), 93-109.

Vasilevskiy, A., \& Siddiqi, K. (2002). Flux maximizing geometric flows. IEEE Transactions on System, Man and Cybernetics Part B, 24(12), 1565-1578.

Wang, Y., \& Lee, S. (1998). Scale-space derived from b-splines. IEEE Transactions on Pattern Analysis and Machine Intelligence, 20(10), 1040-1050. 
Whitaker, R. (1998). A level-set approach to 3-d reconstruction from range data. International Journal of Computer Vision, 29(33), 203-231.

Wong, W., \& Chung, A. (2007). Probabilistic vessel axis tracing and its application to vessel segmentation with stream surfaces and minimum cost paths. Medical Image Analysis, 11(6), 567-587.

Xu, C., \& Prince, J. (1998). Snakes, shapes, and gradient vector flow. IEEE Transactions on Image Processing, 7(3), 359-369.

Yan, P., \& Kassim, A. (2006). Segmentation of volumetric MRA images by using capillary active contour. Medical Image Analysis, 10(3), 317-329.
Yim, P., Choyke, P., \& Summers, R. (2000). Gray-scale skeletonization of small vessels in magnetic resonance angiography. IEEE Transactions on Medical Imaging, 19(6), 568-576.

Zijdenbos, A. P., Dawant, B. M., Margolin, R. A., \& Palmer, A. C. (1994). Morphometric analysis of white matter lesions in MR images: method and validation. IEEE Transactions on Medical Imaging, 13(4), 716-724. 